This item was submitted to Loughborough's Research Repository by the author.

Items in Figshare are protected by copyright, with all rights reserved, unless otherwise indicated.

\title{
Selective strong and weak disposability in efficiency analysis
}

PLEASE CITE THE PUBLISHED VERSION

https://doi.org/10.1016/j.ejor.2019.01.064

PUBLISHER

(C) Elsevier

VERSION

AM (Accepted Manuscript)

PUBLISHER STATEMENT

This paper was accepted for publication in the journal European Journal of Operational Research and the definitive published version is available at https://doi.org/10.1016/j.ejor.2019.01.064

\section{LICENCE}

CC BY-NC-ND 4.0

\section{REPOSITORY RECORD}

Mehdiloo, Mahmood, and Victor Podinovski. 2019. "Selective Strong and Weak Disposability in Efficiency Analysis”. Loughborough University. https://hdl.handle.net/2134/36742. 


\title{
Selective strong and weak disposability in efficiency analysis
}

\author{
Mahmood Mehdiloo ${ }^{\mathrm{a}, *}$, Victor V. Podinovski ${ }^{\mathrm{b}}$ \\ ${ }^{a}$ Department of Mathematics and Applications, University of Mohaghegh Ardabili, Ardabil, Iran \\ ${ }^{b}$ School of Business and Economics, Loughborough University, Loughborough LE11 3TU, UK
}

\begin{abstract}
The conventional constant and variable returns-to-scale models of data envelopment analysis (DEA) incorporate the assumption of strong, or free, disposability. According to this assumption, each input can be increased and each output can be reduced independently of the other measures. In this paper we argue that this assumption may not be suitable in applications in which some inputs or outputs are closely related to each other. Assuming strong disposability of such closely related measures may lead to unrealistic input and output profiles, and result in meaningless efficiency scores. Examples include inputs and outputs that are strongly correlated, represent overlapping measures or situations in which one measure is a subset of another. In this paper we develop production technologies that allow the specification of groups of closely related inputs and outputs which are only jointly weakly disposable. This assumption does not change the existing proportions between the closely related measures in the same group. We demonstrate the usefulness of the suggested approach by computational experiments.
\end{abstract}

Keywords: Data envelopment analysis; Production technology; Weak disposability; Efficiency; Closely related measures

\section{Introduction}

Most theoretical developments and applications of efficiency analysis are based on the assumption of strong, or free, disposability of all inputs and outputs. This assumption is incorporated in the conventional constant and variable returns-to-scale (CRS and VRS) models whose origins can be traced to the earlier literature including the works of Afriat (1972), Shephard (1974a) and Färe, Grosskopf and Logan (1983) - see Førsund and Sarafoglou (2002) for a review. In data envelopment analysis (DEA), these models were firmly established following the seminal works of Charnes, Cooper, and Rhodes (1978) and Banker, Charnes, and Cooper (1984). According to the assumption of strong disposability, if a decision making unit (DMU) is considered to be a member of a production technology, then increasing any of its inputs or reducing any of its outputs keeps the resulting DMU in the technology.

A well-known exception is the treatment of undesirable outputs by the assumption of their joint weak disposability together with the desirable outputs (Färe, Grosskopf, \& Lovell, 1985; Kuosmanen, 2005; Shephard, 1974b). This assumption means that the undesirable and desirable outputs can be reduced simultaneously by the same reduction factor, so that

\footnotetext{
*Corresponding author

Email addresses: m.mehdiloozad@gmail.com (Mahmood Mehdiloo), v.podinovski@lboro.ac.uk (Victor V. Podinovski)
} 
the relative proportions between the resulting outputs are kept constant. A taxonomy of technologies based on the assumption of weak disposability of outputs is explored in detail by Pham and Zelenyuk (2018).

A similar assumption of weak, or ray, disposability of inputs means that the inputs can be increased by the same factor which keeps their relative proportions unchanged. This assumption was modelled in the framework of activity analysis by Shephard (1974a) and was subsequently shown to be useful as a technical tool for testing the input congestion. The idea is to substitute the assumption of strong disposability of the inputs in the VRS technology by their weak disposability. Then, the ratio of the technical efficiency of a DMU evaluated in the VRS technology to its efficiency in the technology based on weak input disposability is interpretable as a measure of input congestion (Byrnes, Färe, Grosskopf, \& Lovell, 1988; Dervaux, Kerstens, \& Vanden Eeckaut, 1998; Färe \& Grosskopf, 1983; Färe et al., 1985; Mehdiloozad \& Podinovski, 2018). ${ }^{1}$

In addition to the above relatively special usages of weak disposability, in this paper we argue that this assumption may be appropriate in a more general modelling setting in which several input or output measures are closely related to each other. This includes technologies in which groups of inputs or outputs should intuitively be in some approximate proportions with respect to each other. In some cases closely related measures may be shown to be statistically strongly correlated. Alternatively, some input or output measures may be overlapping, or one such measure may be a subset of another. In all such cases, assuming strong disposability implies allowing the individual disposal of each input or output independently of its closely related measures, which may result in a DMU with unrealistic or even meaningless proportions between its inputs and outputs.

Below we argue that, in the described scenarios, a safer modelling option may be to assume only weak disposability of the selected groups of closely related inputs and outputs. This allows all closely related inputs to be increased simultaneously by the same factor, thus keeping the relative proportions between the resulting input levels unchanged. Similarly, the closely related outputs can be reduced by the same factor (independent of the factor applied to the inputs), without changing the relative proportions between them. At the same time, we may maintain the assumption of strong disposability with respect to the remaining inputs and outputs. Additionally, as pointed below, in some cases it may also be reasonable to allow strong disposability of some individual inputs and outputs in the weakly disposable subsets. We give a formal definition of these types of (selectively applied) strong and weak disposability in Section 3.

As an illustration, consider the assessment of school efficiency. Many models in this context include a measure of teaching provision, e.g., the number of qualified teachers, and school expenditure (which may or may not include teachers' salaries) as inputs. One may argue that the number of teachers and expenditure are closely related inputs in the sense that the number of teachers reflects the size of the school, and schools with more teachers (and pupils) should normally have higher expenditure. Therefore, it might be unrealistic to assume that the number of teachers as an input could be increased (as in the assumption of strong disposability) without simultaneously increasing the school expenses. However, at the same time, the school expenditure may be increased independently of the teachers and spent, for example, on better school facilities and school trips.

In the above situation it is arguably more realistic to assume joint weak disposability of the number of teachers and school expenditure, while additionally keeping the assumption

\footnotetext{
${ }^{1}$ Färe, Grosskopf and Lovell (1987) use a similar approach to develop nonparametric tests of the input and output disposability.
} 
of strong disposability for the school expenditure only. In other words, we assume that the number of teachers could be increased if accompanied by the increase of the school expenses in the same proportion, but the latter measure could also be increased independently.

On the output side in the same context, we may consider the overall number of pupils and also the number of pupils who have achieved good academic results. Clearly, because the latter output is a subset of the former, assuming strong disposability of all pupils would add hypothetical schools to the technology in which the number of all pupils is smaller than the number of pupils with good results, which is impossible. A seemingly simple solution to this problem may be removing pupils with good results from all pupils. Such model would have pupils who have achieved good results and those who did not as the two outputs. However, this may be problematic because it is not clear whether the latter output is a desirable one. For this reason, it may be more acceptable to keep the original specification of the model in which the overall number of pupils measures the quantity of the services that a school provides, while the number of pupils with good results measures its quality. In this case we can assume joint weak disposability of the two outputs, while simultaneously allowing strong disposability of the number of pupils with good results.

Similarly, in some applications we may prefer to keep some special categories of pupils, such as pupils from disadvantaged backgrounds or pupils with special needs as subsets of the total number of pupils. Subtracting these pupils from the overall number of pupils may not be acceptable for policy reasons. An additional consideration is that different special categories of pupils would typically also be overlapping. Defining the outputs that represent mutually disjoint sets of pupils (such as the pupils with good academic results and with special needs, and not from a disadvantaged background) may lead to a very large number of outputs or simply be impossible because of the lack of information needed for this. It is clear that the assumption of strong disposability of the above outputs may be problematic because of the overlapping of the different categories of pupils that cannot therefore be changed independently of each other. In such situations the weaker assumption of their joint weak disposability may be more appropriate.

Outside the school context, closely related inputs and outputs can easily be found in the other sectors. For example, in hospital applications, the number of doctors and nurses may be deemed to be closely related to the hospital expenses. In banking applications, operational expenses may be treated as closely related to the value of deposits. In various applications, the input representing staff with higher qualifications or performing particular tasks could be included as a subset of all staff.

It may be argued that, in all described situations, changing the assumption of strong disposability in the VRS model to weak disposability with respect to some groups of inputs and outputs does not change the (strongly) efficient frontier of the technology. (We also formally prove this in our paper.) This means that the efficient targets of the inefficient DMUs would still be found on the same efficient frontier, regardless of the type of disposability assumed. However, the Farrell frontier (defined as the set of technically efficient DMUs in the input or output orientations) is generally different under the assumptions of strong and weak disposability, which in turn impacts the efficiency rating of the DMUs projected on it. Therefore, the choice between the assumptions of strong and weak disposability may affect the efficiency rating of DMUs, which is further propagated into various productivity indexes, including measures of productivity change.

In this paper we develop a production technology that allows both assumptions of strong and weak disposability to be made selectively with respect to specific subsets of inputs and outputs. This development addresses the need to take into account groups of closely related inputs and outputs discussed above. 
The proposed technology can be seen as a generalization of the convex technology developed by Mehdiloozad and Podinovski (2018) in two respects. First, in the latter technology, only a subset (or several disjoint subsets) of inputs could be assumed to be weakly disposable. In contrast, the new proposed technology allows simultaneous treatment of both input and output subsets as being weakly disposable, independent of each other. Second, the new technology allows some of the inputs and outputs in the weakly disposable subsets to be additionally treated as strongly disposable, which was not considered in the previous development. (An example of such input is the school expenditure discussed above.)

The new technology may also be loosely viewed as a generalization of the weakly disposable technology of Kuosmanen (2005) which allows joint weak disposability of all good and bad outputs. This comparison is not perfect because the efficient frontier of the technology of Kuosmanen is defined by the maximization of the good outputs and the minimization of the bad outputs, as opposed to the maximization of all outputs in the conventional setting. Mathematically, the statement of the technology of Kuosmanen is a special case of the statement of the general technology proposed in our paper, in which all inputs are assumed to be strongly disposable, and weak disposability is exhibited only on the output side. ${ }^{2}$

We formally derive the new technology from a set of production axioms and obtain its operational statement. The input and output-oriented radial DEA models based on this technology are stated as linear programs. We further consider the evaluation of returns to scale (RTS) in the new technology. We show that this can be accomplished by analyzing the set of dual optimal solutions, similar to the case of the VRS technology. We also consider a more general case in which several groups of inputs and outputs are closely related (within the same group), and develop a model of production technology that addresses this scenario.

In order to demonstrate the usefulness of the suggested new technology, we provide a computational experiment with simulated data in the context of school assessment. The results highlight the difference between the standard VRS model and the models based on a combination of assumptions of strong and weak disposability.

We proceed as follows. In Section 2, we introduce notation and briefly outline the axiomatic approach to the definition of technology which is further used in this paper. In Section 3, we state the axioms of selective strong and weak disposability that are used as the basis of the new technology. In Section 4, we formally derive the new technology based on a combination of disposability assumptions. In Section 5, we obtain the input and output-oriented linear programs based on the new technology, and also consider non-radial models. In Section 6, we develop the notion of one-sided scale elasticity and RTS in the new technology. In Section 7, we obtain a further extension to the case with several groups of closely related inputs and outputs. In Section 8, we consider a computational experiment that illustrates the usefulness of the suggested approach. Section 9 contains concluding remarks and suggestions for future research.

\section{Preliminaries}

\subsection{Notation}

Let $\mathbb{R}^{d}$ denote the $d$-dimensional Euclidean space, and let $\mathbb{R}_{+}^{d}$ denote its nonnegative orthant. We denote sets by uppercase calligraphic letters and vectors by lowercase boldface

\footnotetext{
${ }^{2}$ Another approach to the treatment of closely related inputs and outputs is considered by Tone and Tsutsui (2011). Their model is based on the standard CRS technology of Charnes et al. (1978) and its objective function is a variant of the slack-based measure of Tone (2001). This model is further extended to the setting with undesirable outputs.
} 
letters. All vectors are considered to be column vectors. The transposition of a vector is indicated by superscript $T$. We use bold notation $\mathbf{0}$ and $\mathbf{1}$ to denote the vectors all components of which are equal to 0 and 1, respectively. The dimensions of these vectors are clear from the context in which they are used. For vectors $\mathbf{a}, \mathbf{b} \in \mathbb{R}^{d}$, the inequality $\mathbf{a} \geq \mathbf{b}$ $(\mathbf{a}>\mathbf{b})$ means that $a_{i} \geq b_{i}\left(a_{i}>b_{i}\right)$, for all $i=1, \ldots, d$.

Let $\mathcal{T}$ be a production technology with $m \geq 1$ inputs and $s \geq 1$ outputs. Denote $\mathcal{I}$ the set of inputs and $\mathcal{O}$ the set of outputs. Each element $(\mathbf{x}, \mathbf{y}) \in \mathcal{T}$ is referred to as a decision making units (DMU), where $\mathbf{x}=\left(x_{1}, \ldots, x_{m}\right)^{T}$ and $\mathbf{y}=\left(y_{1}, \ldots, y_{s}\right)^{T}$ are its vectors of inputs and outputs, respectively.

Technology $\mathcal{T}$ includes all DMUs $(\mathbf{x}, \mathbf{y})$ such that $\mathbf{y}$ can be produced from $\mathbf{x}$ :

$$
\mathcal{T}=\left\{(\mathbf{x}, \mathbf{y}) \in \mathbb{R}_{+}^{m} \times \mathbb{R}_{+}^{s} \mid \mathbf{x} \text { can produce } \mathbf{y}\right\}
$$

Let there be $n$ observed DMUs $\left(\mathbf{x}_{j}, \mathbf{y}_{j}\right), j \in \mathcal{J}=\{1, \ldots, n\}$. We denote $\left(\mathbf{x}_{o}, \mathbf{y}_{o}\right)$ the DMU under the evaluation. It can be one of the observed DMUs or, more generally, any DMU in technology $\mathcal{T}$.

We assume that all observed DMUs and DMU $\left(\mathbf{x}_{o}, \mathbf{y}_{o}\right)$ have at least one nonzero input and one nonzero output, i.e., $\mathbf{x}_{j} \neq \mathbf{0}$ and $\mathbf{y}_{j} \neq \mathbf{0}$, for all $j \in \mathcal{J}$, and $\mathbf{x}_{o} \neq \mathbf{0}$ and $\mathbf{y}_{o} \neq \mathbf{0}$.

\subsection{An axiomatic approach to the definition of a technology}

In this paper we employ the axiomatic approach to the definition of production technologies. According to this approach, a production technology $\mathcal{T}$ is defined by particular set of axioms, or properties (see, e.g., Färe, Grosskopf, \& Lovell, 1983, 1985, 1994). We also employ the minimum extrapolation principle (Banker et al., 1984), according to which the technology $\mathcal{T}$ is formally defined as the intersection of all technologies that satisfy the stated axioms. An additional check is required to verify that such intersection itself satisfies all the axioms (which is not always $\operatorname{true}^{3}$ ). If it does, $\mathcal{T}$ is the smallest technology that satisfies the stated axioms.

The use of the minimum extrapolation principle guarantees that $\mathcal{T}$ contains only those DMUs that are needed to satisfy the stated axioms and does not contain any additional arbitrary DMUs that cannot be explained by the axioms.

As an illustration, consider the following three axioms used in the definition of the VRS technology.

Axiom IO (Inclusion of Observations) $\left(\mathbf{x}_{j}, \mathbf{y}_{j}\right) \in \mathcal{T}$, for all $j \in \mathcal{J}$.

Axiom SDIO (Strong Disposability of Inputs and Outputs) If $(\mathbf{x}, \mathbf{y}) \in \mathcal{T}$, then $(\tilde{\mathbf{x}}, \tilde{\mathbf{y}}) \in \mathcal{T}$ for all $\tilde{\mathbf{x}} \geq \mathbf{x}$ and $\mathbf{0} \leq \tilde{\mathbf{y}} \leq \mathbf{y}$.

Axiom CT (Convexity of Technology) $\mathcal{T}$ is a convex set.

Banker et al. (1984) formally derive the VRS technology from the above axioms using the minimum extrapolation principle. They prove that the VRS technology can be stated in the following explicit form:

$$
\mathcal{T}_{\text {VRS }}=\left\{(\mathbf{x}, \mathbf{y}) \in \mathbb{R}_{+}^{m} \times \mathbb{R}_{+}^{s} \mid \exists \boldsymbol{\lambda} \in \mathbb{R}^{n}: \sum_{j \in \mathcal{J}} \lambda_{j} \mathbf{x}_{j} \leq \mathbf{x}, \sum_{j \in \mathcal{J}} \lambda_{j} \mathbf{y}_{j} \geq \mathbf{y}, \mathbf{1}^{T} \boldsymbol{\lambda}=1, \boldsymbol{\lambda} \geq \mathbf{0}\right\} .
$$

It is worth noting that there exist many other technologies that satisfy the above three axioms, for example the CRS technology of Charnes et al. (1978). However, the VRS

\footnotetext{
${ }^{3}$ For example, the intersection of all $S$-shaped technologies that include a finite set of observed DMUs is not an $S$-shaped technology, so the smallest $S$-shaped technology is undefined.
} 
technology is the smallest among all such technologies, and any additional DMUs to those incorporated in the VRS technology would not follow from the above axioms.

A standard additional requirement to any technology $\mathcal{T}$ is that it is a closed set:

Axiom $\mathbf{C}$ (Closedness) $\mathcal{T}$ is a closed set.

It is straightforward to prove that technology $\mathcal{T}_{\text {VRS }}$ satisfies Axiom $\mathrm{C}$. This means that Axioms IO, SDIO and CT imply Axiom $\mathrm{C}$ and make it redundant and unnecessary in the definition of the VRS technology. However, Axiom $\mathrm{C}$ does not always follow from the other axioms assumed in the definition of an arbitrary technology $\mathcal{T}$, in which case $\mathcal{T}$ may not necessarily be a closed set. In particular, such situation arises in the development of the new technology with closely related inputs and outputs in this paper. This means that we have to postulate Axiom $\mathrm{C}$ explicitly for the correct axiomatic definition of the new technology.

\section{Selective strong and weak disposability}

Consider the following decomposition of the set of all inputs $\mathcal{I}$ and the set of all outputs $\mathcal{O}$ defined in Section 2.1 into the mutually disjoint subsets:

$$
\mathcal{I}=\mathcal{I}^{S} \cup \mathcal{I}^{W} \cup \mathcal{I}^{S W}, \quad \mathcal{O}=\mathcal{O}^{S} \cup \mathcal{O}^{W} \cup \mathcal{O}^{S W}
$$

We assume that the inputs in the union of sets $\mathcal{I}^{W} \cup \mathcal{I}^{S W}$ are closely related to each other and are therefore jointly weakly disposable, i.e., can simultaneously be increased in the same proportion $\rho \geq 1$ as a single group. In addition, each of the inputs in the union of sets $\mathcal{I}^{S} \cup \mathcal{I}^{S W}$ is strongly disposable. Consequently, the subset $\mathcal{I}^{S W}$ includes those inputs that are both individually strongly disposable and also weakly disposable as a part of the group $\mathcal{I}^{W} \cup \mathcal{I}^{S W}$.

Two special cases of the decomposition of the input set $\mathcal{I}$ in (2) have been explored in the literature. In the standard setting used by Shephard (1974a) and Färe and Grosskopf (1983), it is assumed that all inputs are jointly weakly disposable, i.e., $\mathcal{I}=\mathcal{I}^{W}$ and $\mathcal{I}^{S}=\mathcal{I}^{S W}=\varnothing$. The more general scenario in which $\mathcal{I}=\mathcal{I}^{S} \cup \mathcal{I}^{W}$ and $\mathcal{I}^{S W}=\varnothing$ was considered by Byrnes et al. (1988) for the purposes of identifying the subset of inputs that exhibits congestion. In this case, it is assumed that the inputs from the subset $\mathcal{I}^{S}$ are strongly disposable and that the inputs from the subset $\mathcal{I}^{W}$ are only weakly disposable, i.e., can simultaneously be increased in an arbitrary proportion $\rho \geq 1$ as a group, but not individually. Mehdiloozad and Podinovski (2018) point that the same setting may also be used as a general modelling assumption, outside the congestion measuring framework.

The novelty of the input decomposition in (2) is that it allows the third term $\mathcal{I}^{S W}$. This term is needed for the modelling of the inputs that play a dual role with regard to the weak and strong disposability characterization, an example of which, as discussed in Section 1, is the school expenditure. Such inputs need to be included in the subset of jointly weakly disposable inputs to make their simultaneous increase by the scaling parameter $\rho \geq 1$ meaningful. However, this single characterization would be incomplete because such inputs are also individually strongly disposable, independently of the other inputs. This dual role of the inputs in the subset $\mathcal{I}^{S W}$ is reflected in the new axioms of weak and strong disposability presented below, and also requires their different mathematical treatment in the models of technology based on these axioms.

Similarly, the outputs in $\mathcal{O}^{W} \cup \mathcal{O}^{S W}$ are closely related and are jointly weakly disposable as a group, i.e., can simultaneously be reduced by the same factor $\sigma \in[0,1]$. The outputs in $\mathcal{O}^{S} \cup \mathcal{O}^{S W}$ are also strongly disposable. Consequently, the outputs in $\mathcal{O}^{S W}$ are individually strongly disposable and are also in the weakly disposable group of outputs $\mathcal{O}^{W} \cup \mathcal{O}^{S W}$. 
A special case of the decomposition of the output set $\mathcal{O}$ specified in (2) is used in the Shephard technology with undesirable outputs (Färe et al., 1985; Shephard, 1974b) and it modification developed by Kuosmanen (2005). In these models, it is assumed that all desirable and undesirable outputs are jointly weakly disposable, whereas the desirable outputs are also strongly disposable. This characterization corresponds to the output decomposition (2) in which the set $\mathcal{O}^{W}$ includes all undesirable outputs and the set $\mathcal{O}^{S W}$ includes all desirable outputs. Additionally, the decomposition (2) allows for the third subset $\mathcal{O}^{S}$ of the outputs that are strongly disposable. In contrast with the subset $\mathcal{O}^{S W}$, the reduction of outputs in $\mathcal{O}^{S}$ is not required when the outputs in the set $\mathcal{O}^{W} \cup \mathcal{O}^{S W}$ are reduced with the same factor $\sigma \in[0,1]$.

Any of the three subsets of inputs $\mathcal{I}^{S}, \mathcal{I}^{W}$ and $\mathcal{I}^{S W}$, but not all of them, may be empty sets. Note that the situation in which $\mathcal{I}^{W}=\varnothing$ and $\mathcal{I}^{S W} \neq \varnothing$ is formally allowed. However, in this case, it would be intuitively clearer to reclassify all inputs in the set $\mathcal{I}^{S W}$ as strongly disposable and incorporate them in the set $\mathcal{I}^{S}$ (because the property of weak disposability of a group of inputs is redundant if all inputs in the group are strongly disposable). The models developed below are valid for either specification. The same is true with respect to the output sets.

In line with the decomposition (2), each DMU (x, y) can be stated as follows:

$$
(\mathbf{x}, \mathbf{y}) \equiv\left(\mathbf{x}^{S}, \mathbf{x}^{W}, \mathbf{x}^{S W}, \mathbf{y}^{S}, \mathbf{y}^{W}, \mathbf{y}^{S W}\right)
$$

where each individual subvector includes the inputs or outputs from the corresponding subset in (2).

The following two axioms state formally the assumptions of strong and weak disposability made with respect to the selected subsets of inputs and outputs.

Axiom SSD (Selective Strong Disposability) If $(\mathbf{x}, \mathbf{y}) \in \mathcal{T}$, then

$$
\left(\tilde{\mathbf{x}}^{S}, \mathbf{x}^{W}, \tilde{\mathbf{x}}^{S W}, \tilde{\mathbf{y}}^{S}, \mathbf{y}^{W}, \tilde{\mathbf{y}}^{S W}\right) \in \mathcal{T}
$$

for all $\tilde{\mathbf{x}}^{S} \geq \mathbf{x}^{S}, \tilde{\mathbf{x}}^{S W} \geq \mathbf{x}^{S W}, \mathbf{0} \leq \tilde{\mathbf{y}}^{S} \leq \mathbf{y}^{S}$ and $\mathbf{0} \leq \tilde{\mathbf{y}}^{S W} \leq \mathbf{y}^{S W}$.

According to Axiom SSD, for any $\operatorname{DMU}(\mathbf{x}, \mathbf{y}) \in \mathcal{T}$, an arbitrary increase of its individual inputs in the subvectors $\mathbf{x}^{S}$ and $\mathbf{x}^{S W}$, and an arbitrary reduction of its outputs in the subvectors $\mathbf{y}^{S}$ and $\mathbf{y}^{S W}$ (as long as these outputs remain nonnegative) keeps the resulting DMU in technology $\mathcal{T}$.

Axiom SWD (Selective Weak Disposability) If $(\mathbf{x}, \mathbf{y}) \in \mathcal{T}$, then

$$
\left(\mathbf{x}^{S}, \rho \mathbf{x}^{W}, \rho \mathbf{x}^{S W}, \mathbf{y}^{S}, \sigma \mathbf{y}^{W}, \sigma \mathbf{y}^{S W}\right) \in \mathcal{T},
$$

for all $\rho \geq 1$ and $0 \leq \sigma \leq 1$.

According to Axiom SWD, for any $\operatorname{DMU}(\mathbf{x}, \mathbf{y}) \in \mathcal{T}$, increasing its input subvectors $\mathbf{x}^{W}$ and $\mathbf{x}^{S W}$ by a factor $\rho \geq 1$, and reducing its output subvectors $\mathbf{y}^{W}$ and $\mathbf{y}^{S W}$ by a factor $\sigma \in[0,1]$ keeps the resulting DMU in technology $\mathcal{T}$.

It is clear that, if technology $\mathcal{T}$ satisfies the standard strong disposability Axiom SDIO stated in the previous section, then $\mathcal{T}$ also satisfies both Axioms SSD and SWD, but the converse is obviously not generally true. Therefore, the two Axioms SSD and SWD together represent a weaker assumption about technology $\mathcal{T}$ than the single standard Axiom SDIO.

Example 1. Suppose that DMU $A$ in Table 1 is a member of some technology $\mathcal{T}$ that satisfies Axioms SSD and SWD. Its three inputs and three outputs are denoted in accordance with (2). 
Table 1: DMUs in Example 1.

\begin{tabular}{ccccccc}
\hline DMU & $x_{1}^{S}$ & $x_{2}^{W}$ & $x_{2}^{S W}$ & $y_{1}^{S}$ & $y_{2}^{W}$ & $y_{3}^{S W}$ \\
\hline$A$ & 10 & 10 & 10 & 10 & 10 & 10 \\
$B$ & 10 & 15 & 15 & 10 & 8 & 8 \\
$C$ & 20 & 15 & 25 & 5 & 8 & 3 \\
$D$ & 20 & 25 & 15 & 5 & 3 & 8 \\
\hline
\end{tabular}

Applying Axiom SWD with $\rho=1.5$ and $\sigma=0.8$, we obtain DMU $B$. Because $\mathcal{T}$ satisfies Axiom SWD, we have $B \in \mathcal{T}$. Furthermore, because the inputs $x_{1}^{S}$ and $x_{3}^{S W}$ and the outputs $y_{1}^{S}$ and $y_{3}^{S W}$ are strongly disposable, by Axiom SSD, DMU $C \in \mathcal{T}$.

Note that DMU $D$ cannot be obtained from the DMU $A$ by Axioms SSD and SWD.

\section{The hybrid disposable technology}

In this section we define the production technology $\mathcal{T}_{\text {VRS }}^{\mathrm{HD}}$ which is an analogue of the VRS technology $\mathcal{T}_{\text {VRS }}$ obtained by replacing the assumption of strong disposability by the axioms of selective strong and weak disposability. This technology is referred to as the hybrid disposable (HD) technology. It is suitable for the modelling of groups of closely related inputs and outputs.

We introduce technology $\mathcal{T}_{\text {VRS }}^{\mathrm{HD}}$ in two steps. First, we define the smallest technology $\tilde{\mathcal{T}}$ that satisfies the same axioms as the VRS technology except for the axiom of strong disposability, which we replace by the weaker Axioms SSD and SWD. The resulting technology can be stated in an intuitively clear nonlinear mathematical form that allows a simple interpretation. However, it turns out that technology $\tilde{\mathcal{T}}$ is generally not a closed set and, consequently, plays only an intermediate role in the development of the HD technology.

In the second step, we obtain technology $\mathcal{T}_{\text {VRS }}^{\mathrm{HD}}$ as the closure of the intermediate technology $\tilde{\mathcal{T}}$. We also state technology $\mathcal{T}_{\text {VRS }}^{\mathrm{HD}}$ in an equivalent linear form.

\subsection{An intermediate not closed HD technology}

Replacing the axiom of strong disposability of the VRS technology (Axiom SDIO) by the weaker Axioms SSD and SWD, leads to the following definition.

Definition 4.1. Technology $\tilde{\mathcal{T}}$ is the intersection of all technologies $\mathcal{T} \subset \mathbb{R}_{+}^{m} \times \mathbb{R}_{+}^{s}$ that satisfy Axioms IO, CT, SSD and SWD.

It is straightforward to prove that technology $\tilde{\mathcal{T}}$ itself satisfies Axioms IO, CT, SSD and SWD and is, therefore, the smallest technology that satisfies them. The next result states technology $\tilde{\mathcal{T}}$ in an explicit form. 
Theorem 4.1. Technology $\tilde{\mathcal{T}}$ is stated as follows:

$$
\begin{aligned}
\tilde{\mathcal{T}}=\left\{(\mathbf{x}, \mathbf{y}) \in \mathbb{R}_{+}^{m} \times \mathbb{R}_{+}^{s} \mid\right. & \exists \boldsymbol{\lambda}, \boldsymbol{\rho}, \boldsymbol{\sigma} \in \mathbb{R}^{n}: \\
& \sum_{j \in \mathcal{J}} \lambda_{j} \mathbf{x}_{j}^{S} \leq \mathbf{x}^{S}, \\
& \sum_{j \in \mathcal{J}} \lambda_{j} \rho_{j} \mathbf{x}_{j}^{W}=\mathbf{x}^{W}, \\
& \sum_{j \in \mathcal{J}} \lambda_{j} \rho_{j} \mathbf{x}_{j}^{S W} \leq \mathbf{x}^{S W}, \\
& \sum_{j \in \mathcal{J}} \lambda_{j} \mathbf{y}_{j}^{S} \geq \mathbf{y}^{S}, \\
& \sum_{j \in \mathcal{J}} \lambda_{j} \sigma_{j} \mathbf{y}_{j}^{W}=\mathbf{y}^{W}, \\
& \sum_{j \in \mathcal{J}} \lambda_{j} \sigma_{j} \mathbf{y}_{j}^{S W} \geq \mathbf{y}^{S W}, \\
& \left.\mathbf{1}^{T} \boldsymbol{\lambda}=1, \boldsymbol{\lambda} \geq \mathbf{0}, \boldsymbol{\rho} \geq \mathbf{1}, \mathbf{0} \leq \boldsymbol{\sigma} \leq \mathbf{1}\right\} .
\end{aligned}
$$

The proofs of Theorem 4.1 and the other statements are given in Appendix A.

The statement $(3)$ of technology $\tilde{\mathcal{T}}$ allows its straightforward interpretation. Indeed, for each $j \in \mathcal{J}$, consider the observed $\operatorname{DMU}\left(\mathbf{x}_{j}, \mathbf{y}_{j}\right)$ on the left-hand side of $(3)$. In line with Axiom SWD, its weakly disposable inputs are proportionately increased by the multiplier $\rho_{j}$ and weakly disposable outputs are proportionally reduced by the multiplier $\sigma_{j}$. By Axiom SWD, the resulting DMU is in technology $\tilde{\mathcal{T}}$. By Axiom CT, the convex combination of the DMUs taken with the corresponding weights $\lambda_{j}, j \in \mathcal{J}$, on the left-hand side of $(3)$ is in $\tilde{\mathcal{T}}$. Finally, by Axiom SSD, the DMU $(\mathbf{x}, \mathbf{y})$ on the right-hand side of $(3)$ is in $\tilde{\mathcal{T}}$.

Interestingly, $\tilde{\mathcal{T}}$ is generally not a closed technology. We show this in Example 2 after the development of the closed HD technology. The next theorem highlights a special case in which $\tilde{\mathcal{T}}$ is a closed set.

Theorem 4.2. Let all inputs be strongly disposable, i.e., let $\mathcal{I}^{\mathcal{S}}=\mathcal{I}$. Then $\tilde{\mathcal{T}}$ is a polyhedral, and therefore closed, technology.

As an illustration to the above theorem, consider the technology of Kuosmanen (2005) developed for the treatment of undesirable outputs, which we denote $\mathcal{T}^{\mathrm{K}}$. In this technology, all inputs are assumed to be strongly disposable, and its nonlinear statement is a special case of (3). By theorem 4.2, $\mathcal{T}^{\mathrm{K}}$ is a closed polyhedral technology, which was independently established by Podinovski and Kuosmanen (2011).

In the next subsection we redefine the HD technology by incorporating the additional Axiom C. This not only defines a closed HD technology but also leads to a more usable statement of this technology in the form of linear equalities and inequalities.

\subsection{The closed HD technology}

In the previous subsection we demonstrated that, simply replacing the axiom of strong disposability in the definition of the VRS technology by its two weaker analogues in order to model closely related inputs and outputs results in technology $\tilde{\mathcal{T}}$ that is generally not a closed set. In other words, Axioms IO, CT, SSD and SWD do not imply Axiom C, and the latter has to be required explicitly in the definition of technology. 
Definition 4.2. The closed convex HD technology $\mathcal{T}_{\mathrm{VRS}}^{\mathrm{HD}}$ is the intersection of all technologies $\mathcal{T} \subset \mathbb{R}_{+}^{m} \times \mathbb{R}_{+}^{s}$ that satisfy Axioms IO, CT, SSD, SWD and C.

It is straightforward to prove that technology $\mathcal{T}_{\text {VRS }}^{\mathrm{HD}}$ itself satisfies all Axioms IO, CT, SSD, SWD and C and is, therefore, the smallest technology that satisfies them.

It is clear that technology $\mathcal{T}_{\text {VRS }}$ also satisfies Axioms IO, CT, SSD, SWD and C. (In particular, it satisfies the stronger Axiom SDIO which implies Axioms SSD and SWD.) Therefore, we always have the following embedding:

$$
\mathcal{T}_{\mathrm{VRS}}^{\mathrm{HD}} \subseteq \mathcal{T}_{\mathrm{VRS}}
$$

The following result shows that technology $\mathcal{T}_{\text {VRS }}^{\mathrm{HD}}$ coincides with the closure of technology $\tilde{\mathcal{T}}$, denoted $\operatorname{cl}(\tilde{\mathcal{T}})$. This means that technology $\mathcal{T}_{\text {VRS }}^{\text {HD }}$ is obtained from technology $\tilde{\mathcal{T}}$ by adding all its "missing" boundary points.

Theorem 4.3. The following equality is true: $\mathcal{T}_{\text {VRS }}^{\mathrm{HD}}=\operatorname{cl}(\tilde{\mathcal{T}})$.

To obtain an explicit statement of technology $\mathcal{T}_{\text {VRS }}^{\text {HD }}$, consider the following substitution of variables in the statement (3):

$$
\mu_{j}=\lambda_{j}\left(\rho_{j}-1\right), \quad \nu_{j}=\lambda_{j}\left(1-\sigma_{j}\right), \quad \forall j \in \mathcal{J},
$$

or, equivalently,

$$
\lambda_{j} \rho_{j}=\lambda_{j}+\mu_{j}, \quad \lambda_{j} \sigma_{j}=\lambda_{j}-\nu_{j}, \quad \forall j \in \mathcal{J} .
$$

The above transformation restates any DMU in technology $\tilde{\mathcal{T}}$, which is expressed in terms of vectors $\boldsymbol{\lambda}, \boldsymbol{\rho}$ and $\boldsymbol{\sigma}$ in (3), by its statement in (7) given below in terms of vectors $\boldsymbol{\lambda}, \boldsymbol{\mu}$ and $\boldsymbol{\nu}$. However, this is not an equivalent transformation. This is because, as the next result shows, transformation (5) slightly enlarges technology $\tilde{\mathcal{T}}$ which is just enough to obtain its closure $\mathcal{T}_{\text {VRS }}^{\text {HD }}{ }^{4}$

Theorem 4.4. Technology $\mathcal{T}_{\mathrm{VRS}}^{\mathrm{HD}}$ is stated as follows:

$$
\begin{aligned}
\mathcal{T}_{\mathrm{VRS}}^{\mathrm{HD}}=\left\{(\mathbf{x}, \mathbf{y}) \in \mathbb{R}_{+}^{m} \times \mathbb{R}_{+}^{s} \mid\right. & \exists \boldsymbol{\lambda}, \boldsymbol{\mu}, \boldsymbol{\nu} \in \mathbb{R}^{n}: \\
& \sum_{j \in \mathcal{J}} \lambda_{j} \mathbf{x}_{j}^{S} \leq \mathbf{x}^{S}, \\
& \sum_{j \in \mathcal{J}}\left(\lambda_{j}+\mu_{j}\right) \mathbf{x}_{j}^{W}=\mathbf{x}^{W}, \\
& \sum_{j \in \mathcal{J}}\left(\lambda_{j}+\mu_{j}\right) \mathbf{x}_{j}^{S W} \leq \mathbf{x}^{S W}, \\
& \sum_{j \in \mathcal{J}} \lambda_{j} \mathbf{y}_{j}^{S} \geq \mathbf{y}^{S}, \\
& \sum_{j \in \mathcal{J}}\left(\lambda_{j}-\nu_{j}\right) \mathbf{y}_{j}^{W}=\mathbf{y}^{W}, \\
& \sum_{j \in \mathcal{J}}\left(\lambda_{j}-\nu_{j}\right) \mathbf{y}_{j}^{S W} \geq \mathbf{y}^{S W}, \\
& \left.\mathbf{1}^{T} \boldsymbol{\lambda}=1, \boldsymbol{\lambda} \geq \boldsymbol{\nu}, \boldsymbol{\lambda}, \boldsymbol{\mu}, \boldsymbol{\nu} \geq \mathbf{0}\right\} .
\end{aligned}
$$

\footnotetext{
${ }^{4}$ It is possible that a $\operatorname{DMU}(\hat{\mathbf{x}}, \hat{\mathbf{y}}) \in \mathcal{T}_{\text {VRS }}^{\mathrm{HD}}$ satisfies $(7)$ with $\lambda_{j^{\prime}}=\nu_{j^{\prime}}=0$ and $\mu_{j^{\prime}}>0$, for some $j^{\prime} \in \mathcal{J}$. Such DMU $(\hat{\mathbf{x}}, \hat{\mathbf{y}})$ may not be feasible in technology $\tilde{\mathcal{T}}$ defined by $(3)$, because no $\rho_{j^{\prime}}$ satisfies the inverse transformation (5). We discuss this further in Example 2.
} 
Table 2: The data set in Example 2.

\begin{tabular}{cccc}
\hline DMU & $x_{1}$ & $x_{2}$ & $y$ \\
\hline$A$ & 2 & 1 & 1 \\
$B$ & 1 & 3 & 5 \\
\hline
\end{tabular}

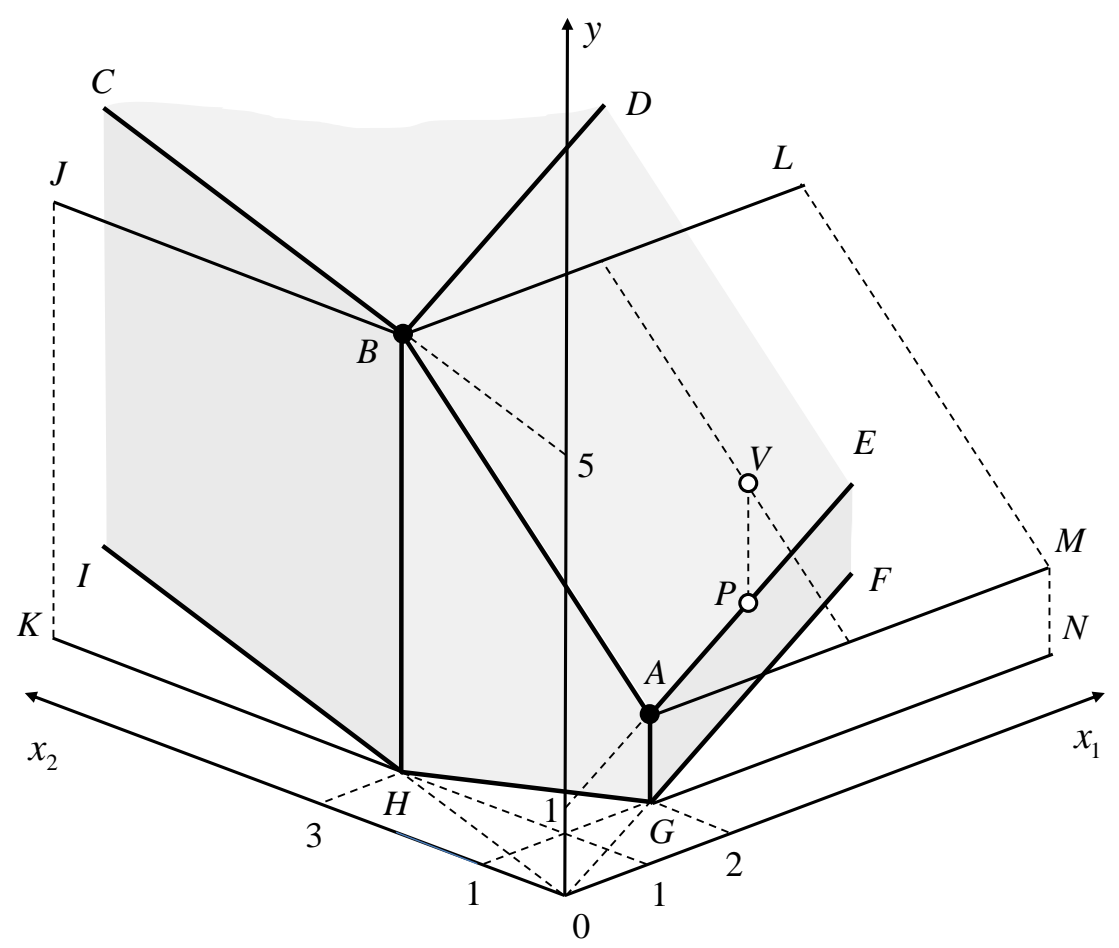

Figure 1: Technology $\mathcal{T}_{\text {VRS }}^{\mathrm{HD}}$ with the superimposed technology $\mathcal{T}_{\text {VRS }}$ generated by DMUs $A$ and $B$.

The following result is important for the returns-to-scale (RTS) characterization of DMUs in technology $\mathcal{T}_{\text {VRS }}^{\text {HD }}$ considered in Section 6.

Theorem 4.5. Technology $\mathcal{T}_{\mathrm{VRS}}^{\mathrm{HD}}$ is a polyhedral set.

As an illustration, consider the following example which is modified from the example given in Mehdiloozad and Podinovski (2018).

Example 2. Consider technology $\mathcal{T}_{\text {VRS }}^{\text {HD }}$ generated by the DMUs $A$ and $B$ as in Table 2 . We assume that the inputs $x_{1}$ and $x_{2}$ are jointly weakly disposable and that the single output is strongly disposable. Fig. 1 shows technology $\mathcal{T}_{\text {VRS }}^{\mathrm{HD}}$ as the shaded polyhedral set. ${ }^{5}$ For reference, we have superimposed the standard technology $\mathcal{T}_{\text {VRS }}$. It includes the rays $B L$, $A M, G N, B J$ and $H K$, and incorporates technology $\mathcal{T}_{\text {VRS }}^{\mathrm{HD}}$ as a subset.

As shown by Mehdiloozad and Podinovski (2018), the intermediate not closed HD technology $\tilde{\mathcal{T}}$ defined in the previous subsection is the same as technology $\mathcal{T}_{\text {VRS }}^{\text {HD }}$ with the following

\footnotetext{
${ }^{5}$ For a detailed explanation of the construction of this technology, see Mehdiloozad and Podinovski (2018), where it is referred to as technology $\mathcal{T}^{\text {WID }}$.
} 
exception: $\tilde{\mathcal{T}}$ does not include the relative interiors of the half-line $B D$ and the facet $C B D$ (but it does include the ray $B C){ }^{6}$

Several special cases of technology $\mathcal{T}_{\text {VRS }}^{\mathrm{HD}}$ are worth highlighting. If all inputs and outputs are strongly disposable, i.e., if $\mathcal{I}^{S}=\mathcal{I}$ and $\mathcal{O}^{S}=\mathcal{O}$, technology $\mathcal{T}_{\text {VRS }}^{\text {HD }}$ is the conventional technology $\mathcal{T}_{\text {VRS }}$. If all inputs are weakly disposable and all outputs are strongly disposable, i.e., if $\mathcal{I}^{W}=\mathcal{I}$ and $\mathcal{O}^{S}=\mathcal{O}, \mathcal{T}_{\text {VRS }}^{\text {HD }}$ is the closed convex technology $\mathcal{T}^{\text {WID }}$ developed by Mehdiloozad and Podinovski (2018). This technology was illustrated in Example 2. ${ }^{7}$

Interestingly, the linearized statement of the technology $\mathcal{T}^{\mathrm{K}}$ developed by Kuosmanen (2005) for the treatment of undesirable outputs is identical to the statement (7) if all inputs and desirable outputs are strongly disposable, and all (desirable and undesirable outputs) are jointly weakly disposable. However, although technology $\mathcal{T}^{\mathrm{K}}$ is mathematically the same as $\mathcal{T}$ VRS , their efficient frontiers are defined differently, and the assessment of efficiency of a numerically identical DMU in the two technologies would generally produce different results. This is because, in technology $\mathcal{T}^{\mathrm{K}}$, efficient production is defined by the minimization of the undesirable outputs and the maximization of the desirable ones, while the efficient frontier of technology $\mathcal{T}_{\text {VRS }}^{\text {HD }}$ is defined by the maximization of all outputs (see Section 4.3 below). ${ }^{8}$

\subsection{Strong and weak efficiency}

In this subsection we prove a useful theorem establishing the relationship between the strong and weak efficient frontiers of technologies $\mathcal{T}_{\text {VRS }}^{\text {HD }}$ and $\mathcal{T}_{\text {VRS }}$. We start with the general definitions applicable to any technology $\mathcal{T}$.

Definition 4.3. A DMU $(\mathbf{x}, \mathbf{y}) \in \mathcal{T}$ is strongly efficient if there does not exist a DMU $\left(\mathbf{x}^{\prime}, \mathbf{y}^{\prime}\right) \in \mathcal{T}$, such that $\mathbf{x}^{\prime} \leq \mathbf{x}, \mathbf{y}^{\prime} \geq \mathbf{y}$, and $\left(\mathbf{x}^{\prime}, \mathbf{y}^{\prime}\right) \neq(\mathbf{x}, \mathbf{y})$.

Definition 4.4. A DMU $(\mathbf{x}, \mathbf{y}) \in \mathcal{T}$ is weakly efficient if there does not exist a DMU $\left(\mathbf{x}^{\prime}, \mathbf{y}^{\prime}\right) \in \mathcal{T}$, such that $\mathbf{x}^{\prime}<\mathbf{x}$ and $\mathbf{y}^{\prime}>\mathbf{y}$.

Let $\mathcal{E}_{\mathrm{HD}}^{*}$ and $\mathcal{E}_{\mathrm{VRS}}^{*}$ be the sets of all strongly efficient DMUs in technologies $\mathcal{T}_{\mathrm{VRS}}^{\mathrm{HD}}$ and $\mathcal{T}_{\text {VRS }}$, respectively. The sets $\mathcal{E}_{\mathrm{HD}}^{*}$ and $\mathcal{E}_{\mathrm{VRS}}^{*}$ are referred to as the (strong) efficient frontiers of these technologies.

Similarly, let $\mathcal{W}_{\mathrm{HD}}^{*}$ and $\mathcal{W}_{\mathrm{VRS}}^{*}$ be the sets of all weakly efficient DMUs in technologies $\mathcal{T}_{\text {VRS }}^{\mathrm{HD}}$ and $\mathcal{T}_{\text {VRS }}$, respectively. These sets are referred to as the weak efficient frontiers of the corresponding HD and VRS technologies.

Because a strongly efficient DMU is also weakly efficient, we obviously have the embeddings $\mathcal{E}_{\mathrm{HD}}^{*} \subseteq \mathcal{W}_{\mathrm{HD}}^{*}$ and $\mathcal{E}_{\mathrm{VRS}}^{*} \subseteq \mathcal{W}_{\mathrm{VRS}}^{*}$. We also have the following result.

\footnotetext{
${ }^{6}$ Each DMU $(\hat{\mathbf{x}}, \hat{y})$ in these excluded relative interiors satisfies $(7)$ stated in terms of the observed DMUs $A$ and $B$, with some vectors $\hat{\boldsymbol{\lambda}}=\left(\hat{\lambda}_{A}, \hat{\lambda}_{B}\right)^{T}$ and $\hat{\boldsymbol{\mu}}=\left(\hat{\mu}_{A}, \hat{\mu}_{B}\right)^{T}$. (Because the output is strongly disposable, the vector $\hat{\boldsymbol{\nu}}=\left(\hat{\nu}_{A}, \hat{\nu}_{B}\right)^{T}$ is not used.) Then we necessarily have $\hat{\lambda}_{A}=0$ and $\hat{\lambda}_{B}=1$, as otherwise the output $\hat{y}$ is less than the output $\hat{y}_{B}=5$ of DMU $B$, which contradicts the assumption that DMU $(\hat{\mathbf{x}}, \hat{y})$ is located on the facet $C B D$. Note that $\hat{\mu}_{A}>0$, as otherwise the $\operatorname{DMU}(\hat{\mathbf{x}}, \hat{y})$ is located on the half-line $B C$. It is straightforward to prove that the representation $(\hat{\mathbf{x}}, \hat{y})=\left(\left(1+\hat{\mu}_{B}\right) \hat{\mathbf{x}}_{B}+\hat{\mu}_{A} \hat{\mathbf{x}}_{A}, \hat{y}_{B}\right)$ is unique. As shown in Footnote 4 , in this case the inverse transformation $(5)$ is undefined, so $(\hat{\mathbf{x}}, \hat{y}) \notin \tilde{\mathcal{T}}$.

${ }^{7}$ Mehdiloozad and Podinovski (2018) consider a further generalization of technology $\mathcal{T}^{\text {WID }}$ in which a subset of all inputs is strongly disposable, and its complementary subset is weakly disposable, i.e., the case in which $\mathcal{I}^{W} \neq \varnothing, \mathcal{I}^{S} \neq \varnothing, \mathcal{I}^{S W}=\varnothing$ and $\mathcal{O}^{S}=\mathcal{O}$. The resulting technology is also a special case of technology $\mathcal{T}_{\text {VRS }}^{\mathrm{HD}}$.

${ }^{8}$ In Example 4, we consider a graphical illustration of technology $\mathcal{T}_{\text {VRS }}^{\text {HD }}$. The strongly efficient (nondominated) frontier of this technology is the segment $A B$ in Fig. 3. We can also view the same technology as the technology $\mathcal{T}^{\mathrm{K}}$ in which output $y_{1}$ is undesirable and output $y_{2}$ is desirable. In this case the efficient frontier is the broken line $O C B$.
} 
Theorem 4.6. The following relations are true:

(i) $\mathcal{E}_{\mathrm{HD}}^{*}=\mathcal{E}_{\mathrm{VRS}}^{*}$,

(ii) $\mathcal{W}_{\mathrm{HD}}^{*} \subseteq \mathcal{W}_{\mathrm{VRS}}^{*}$.

It is worth highlighting the following interesting implication of the above theorem.

Corollary 4.1. If $\operatorname{DMU}(\mathbf{x}, \mathbf{y})$ is strongly efficient in technology $\mathcal{T}_{\mathrm{VRS}}$, then $(\mathbf{x}, \mathbf{y}) \in \mathcal{T}_{\mathrm{VRS}}^{\mathrm{HD}}$.

The relationship between the strong and weak efficient frontiers established by Theorem 4.6 is illustrated in the input and output dimensions by Examples 3 and 4 below. In particular, in both examples, the embedding established by part (ii) of Theorem 4.6 is proper, i.e., we have $\mathcal{W}_{\mathrm{HD}}^{*} \subseteq \mathcal{W}_{\mathrm{VRS}}^{*}$ and $\mathcal{W}_{\mathrm{HD}}^{*} \neq \mathcal{W}_{\mathrm{VRS}}^{*}$.

Note that Theorem 4.6 is not valid for the technology $\mathcal{T}^{\mathrm{K}}$ of Kuosmanen (2005) discussed above as a special case of technology $\mathcal{T}_{\text {VRS }}^{\mathrm{HD}}$. As shown, this is because the definitions of strong and weak efficiencies in technologies $\mathcal{T}^{\mathrm{K}}$ and $\mathcal{T}_{\text {VRS }}^{\mathrm{HD}}$ are different.

\subsection{Reference technologies}

Let $\mathcal{T}_{\text {VRS }}, \mathcal{T}_{\text {CRS }}, \mathcal{T}_{\text {NIRS }}$ and $\mathcal{T}_{\text {NDRS }}$ be the conventional VRS, CRS, non-increasing and non-decreasing returns-to-scale (NIRS and NDRS) technologies based on the assumption of strong disposability of all inputs and outputs.

Technology $\mathcal{T}_{\text {VRS }}^{\mathrm{HD}}$ may be regarded as an analogue of technology $\mathcal{T}_{\text {VRS }}$ in which the assumption of strong disposability is relaxed and replaced by the weaker axioms of selective strong and weak disposability. Similarly, we may consider the relaxation of the axiom of strong disposability in the definition of technology $\mathcal{T}_{\mathrm{CRS}}$ (Axiom SDIO) by replacing it by Axioms SSD and SWD. This technology, denoted $\mathcal{T}_{\mathrm{CRS}}^{\mathrm{HD}}$, requires a similar axiomatic development which leads to the statement (7) in which the normalizing equality $\mathbf{1}^{T} \boldsymbol{\lambda}=1$ is omitted. Finally, we may define the NIRS and NDRS technologies $\mathcal{T}_{\text {NIRS }}^{\text {HD }}$ and $\mathcal{T}_{\text {NDRS }}^{\text {HD }}$ based on the same relaxed disposability axioms. As follows from the more general results established by Podinovski and Bouzdine-Chameeva (2019), this requires replacing the normalizing equality in (7) by the inequalities $\mathbf{1}^{T} \boldsymbol{\lambda} \leq 1$ and $\mathbf{1}^{T} \boldsymbol{\lambda} \geq 1$, respectively.

Similar to the result obtained in Grosskopf (1986), and extending the embedding (4), it is straightforward to establish the relationship between the above technologies. In order to avoid repetition, we include only the NIRS technologies but omit the NDRS technologies from the next statement. This theorem remains true if the NIRS technologies $\mathcal{T}_{\text {NIRS }}$ and $\mathcal{T}_{\text {NIRS }}^{\text {HD }}$ are replaced by the corresponding NDRS technologies $\mathcal{T}_{\text {NDRS }}$ and $\mathcal{T}_{\text {NDRS }}^{\text {HD }}$

Theorem 4.7. The following embeddings are true:

(i) $\mathcal{T}_{\text {VRS }}^{\mathrm{HD}} \subseteq \mathcal{T}_{\mathrm{NIRS}}^{\mathrm{HD}} \subseteq \mathcal{T}_{\mathrm{CRS}}^{\mathrm{HD}}$;

(ii) $\mathcal{T}_{\text {VRS }}^{\mathrm{HD}} \subseteq \mathcal{T}_{\text {VRS }}, \mathcal{T}_{\text {NIRS }}^{\mathrm{HD}} \subseteq \mathcal{T}_{\mathrm{NIRS}}, \mathcal{T}_{\mathrm{CRS}}^{\mathrm{HD}} \subseteq \mathcal{T}_{\mathrm{CRS}}$

The technologies $\mathcal{T}_{\text {CRS }}^{\mathrm{HD}}, \mathcal{T}_{\text {NIRS }}^{\mathrm{HD}}$ and $\mathcal{T}_{\text {NDRS }}^{\mathrm{HD}}$ may be used as reference technologies for testing RTS in technology $\mathcal{T}_{\text {VRS }}^{\text {HD }}$ by the method developed by Färe et al. (1985) and further modified by Kerstens and Vanden Eeckaut (1999) and Briec, Kerstens, Leleu, and Vanden Eeckaut (2000). We are not pursuing this approach further. Instead, in Section 6 we consider an approach based on the evaluation of scale elasticity in technology $\mathcal{T}_{\text {VRS }}^{\text {HD }}$ wich is known to result in an equivalent RTS characterization to that of the method based on the use of reference technologies. ${ }^{9}$

\footnotetext{
${ }^{9}$ By Theorem 4.5, $\mathcal{T}_{\mathrm{VRS}}^{\mathrm{HD}}$ is a polyhedral and, therefore, convex technology. The equivalence of the two methods for the evaluation of RTS in any convex technology is established by Podinovski (2017).
} 


\section{DEA models based on the HD technology}

Below we consider the evaluation of the input and output radial efficiency of DMU $\left(\mathbf{x}_{o}, \mathbf{y}_{o}\right)$ in technology $\mathcal{T}_{\mathrm{VRS}}^{\mathrm{HD}}$. We also briefly comment on the use of non-radial measures in this technology.

\subsection{The input-oriented model}

Consider the statement of technology $\mathcal{T}_{\text {VRS }}^{\mathrm{HD}}$ obtained in Theorem 4.4. Based on this statement, the input radial efficiency of $\operatorname{DMU}\left(\mathbf{x}_{o}, \mathbf{y}_{o}\right)$ in $\mathcal{T}_{\mathrm{VRS}}^{\mathrm{HD}}$ is the optimal value $\theta_{o}^{\mathrm{HD}}$ of the following model:

$$
\begin{aligned}
& \theta_{o}^{\mathrm{HD}}=\min \quad \theta \\
& \text { subject to } \\
& \sum_{j \in \mathcal{J}} \lambda_{j} \mathbf{x}_{j}^{S} \leq \theta \mathbf{x}_{o}^{S}, \\
& \sum_{j \in \mathcal{J}}\left(\lambda_{j}+\mu_{j}\right) \mathbf{x}_{j}^{W}=\theta \mathbf{x}_{o}^{W}, \\
& \sum_{j \in \mathcal{J}}\left(\lambda_{j}+\mu_{j}\right) \mathbf{x}_{j}^{S W} \leq \theta \mathbf{x}_{o}^{S W}, \\
& \sum_{j \in \mathcal{J}} \lambda_{j} \mathbf{y}_{j}^{S} \geq \mathbf{y}_{o}^{S}, \\
& \sum_{j \in \mathcal{J}}\left(\lambda_{j}-\nu_{j}\right) \mathbf{y}_{j}^{W}=\mathbf{y}_{o}^{W}, \\
& \sum_{j \in \mathcal{J}}\left(\lambda_{j}-\nu_{j}\right) \mathbf{y}_{j}^{S W} \geq \mathbf{y}_{o}^{S W}, \\
& \mathbf{1}^{T} \boldsymbol{\lambda}=1, \\
& \boldsymbol{\lambda}-\boldsymbol{\nu} \geq \mathbf{0}, \\
& \boldsymbol{\lambda}, \boldsymbol{\mu}, \boldsymbol{\nu} \geq \mathbf{0}, \theta \text { sign free. }
\end{aligned}
$$

It is clear that $0<\theta_{o}^{\mathrm{HD}} \leq 1 .{ }^{10}$ Program (8) identifies the input radial projection $\left(\theta_{o}^{\mathrm{HD}} \mathbf{x}_{o}, \mathbf{y}_{o}\right)$ of the assessed DMU on the boundary of technology $\mathcal{T}_{\mathrm{VRS}}^{\mathrm{HD}}$.

As stated in (4), technology $\mathcal{T}_{\text {VRS }}^{\mathrm{HD}}$ is a subset of the VRS technology $\mathcal{T}_{\text {VRS }}$. Therefore, we always have $\theta_{o}^{\mathrm{VRS}} \leq \theta_{o}^{\mathrm{HD}}$, where $\theta_{o}^{\mathrm{VRS}}$ is the input radial efficiency of $\mathrm{DMU}\left(\mathbf{x}_{o}, \mathbf{y}_{o}\right)$ evaluated in the VRS technology. The ratio

$$
\theta_{o}^{\mathrm{B}}=\frac{\theta_{o}^{\mathrm{VRS}}}{\theta_{o}^{\mathrm{HD}}}
$$

may be regarded as a measure of the misspecification bias arising when we use the conventional VRS model in a situation in which the inputs and outputs do not satisfy the standard assumption of strong disposability but instead satisfy its weaker analogues stated by Axioms SSD and SWD.

It is clear that $0<\theta_{o}^{\mathrm{B}} \leq 1$. If $\theta_{o}^{\mathrm{B}}=1$ then the misspecification bias is not observed and we have $\theta_{o}^{\mathrm{HD}}=\theta_{o}^{\mathrm{VRS}}$. In this case the projection $\left(\theta_{o}^{\mathrm{HD}} \mathbf{x}_{o}, \mathbf{y}_{o}\right)$ identified by program (8)

\footnotetext{
${ }^{10}$ Indeed, because DMU $\left(\mathbf{x}_{o}, \mathbf{y}_{o}\right) \in \mathcal{T}_{\text {VRS }}^{\mathrm{HD}}$, it satisfies $(7)$ with some vectors $\hat{\boldsymbol{\lambda}}, \hat{\boldsymbol{\mu}}$ and $\hat{\boldsymbol{\nu}}$. These vectors and $\theta=1$ are a feasible solution of program (8). Therefore, $\theta_{o}^{\mathrm{HD}} \leq 1$. By $(8 \mathrm{~b}), \theta_{o}^{\mathrm{HD}} \geq 0$. Furthermore, as assumed, each observed DMU has at least one strictly positive input and output. If $\theta_{o}^{\mathrm{HD}}=0$, then from (8b)-(8d), $\boldsymbol{\lambda}=\mathbf{0}$, which contradicts (8h). Therefore, $\theta_{o}^{\mathrm{HD}}>0$.
} 
Table 3: The data set in Example 3.

\begin{tabular}{cccc}
\hline DMU & $x_{1}$ & $x_{2}$ & $y$ \\
\hline$A$ & 2 & 1 & 1 \\
$B$ & 1 & 2 & 1 \\
$C$ & 1.5 & 8 & 1 \\
$D$ & 2 & 8 & 1 \\
\hline
\end{tabular}

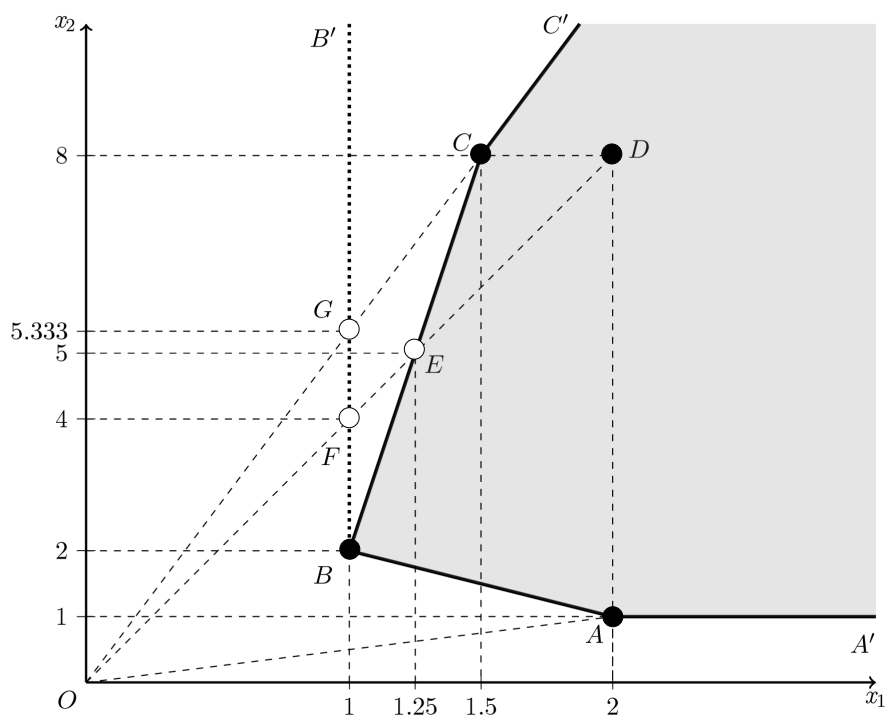

Figure 2: Technology $\mathcal{T}_{\text {VRS }}^{\mathrm{HD}}$ in Example 3.

is located on the (input radial efficient) boundaries of both technologies $\mathcal{T}_{\text {VRS }}^{\mathrm{HD}}$ and $\mathcal{T}_{\text {VRS }}$. Otherwise, if $\theta_{o}^{\mathrm{B}}<1$, the projection $\left(\theta_{o}^{\mathrm{HD}} \mathbf{x}_{o}, \mathbf{y}_{o}\right)$ is located on the boundary of technology $\mathcal{T}_{\text {VRS }}^{\text {HD }}$ but inside the corresponding boundary of technology $\mathcal{T}_{\text {VRS }}$.

As an illustration, consider the following example.

Example 3. Consider the four observed DMUs $A, B, C$ and $D$ shown in Table 3, each of which consumes two inputs to produce one output. Because the output level is the same for all DMUs, we provide an illustration in the input dimensions only (Fig. 2).

Assume that input $x_{1}$ is strongly disposable and that inputs $x_{1}$ and $x_{2}$ are jointly weakly disposable, i.e., using notation as in (2), let $\mathcal{I}^{S}=\varnothing, \mathcal{I}^{W}=\left\{x_{2}\right\}$ and $\mathcal{I}^{S W}=\left\{x_{1}\right\}$. For the stated assumptions, the HD technology $\mathcal{T}_{\text {VRS }}^{\text {HD }}$ is shown (in the input dimensions) as the shaded area $A^{\prime} A B C C^{\prime}$. In comparison, the conventional VRS technology which assumes strong disposability of both inputs, is the larger area defined by its boundary $A^{\prime} A B B^{\prime}$.

As an illustration of Theorem 4.6, note that the strong efficient frontier of both technologies is the segment $A B$. Further note that, the weak efficient frontier of the HD technology (in the two input dimensions, for the fixed level of output $y=1$ ) is $A^{\prime} A B$, which is a proper subset of the weak efficient frontier $A^{\prime} A B B^{\prime}$ of the VRS technology.

Also, in line with the analysis of Färe et al. (1985), define the Farrell frontier as the set of DMUs whose technical (in this example, input radial) efficiency is equal to 1. The Farrell frontier of the VRS technology is its weak efficient frontier $A^{\prime} A B B^{\prime}$. The Farrell frontier of the HD technology is the union of three segments $A^{\prime} A B C$. Note that neither frontier is a 
Table 4: Input radial efficiency and misspecification bias in Example 3.

\begin{tabular}{cccc}
\hline DMU & $\theta_{o}^{\mathrm{VRS}}$ & $\theta_{o}^{\mathrm{HD}}$ & $\theta_{o}^{\mathrm{B}}$ \\
\hline$A$ & 1 & 1 & 1 \\
$B$ & 1 & 1 & 1 \\
$C$ & 0.667 & 1 & 0.667 \\
$D$ & 0.5 & 0.625 & 0.8 \\
\hline
\end{tabular}

subset of the other.

Note that DMU $C$ is input radial efficient in technology $\mathcal{T}_{\text {VRS }}^{\mathrm{HD}}$ but is inefficient in $\mathcal{T}_{\text {VRS }}$. Its projection in the latter technology is DMU $G$. Furthermore, the input radial efficiency of DMU $D$ in technology $\mathcal{T}_{\text {VRS }}^{\mathrm{HD}}$ is measured relative to the segment $B C$ of its boundary. Its projection is DMU $E$ which is located inside the VRS technology (in the input dimensions). The corresponding projection in the VRS technology is DMU $F$.

Table 4 shows the input radial efficiency of each observed DMU in both technologies $\mathcal{T}_{\text {VRS }}$ and $\mathcal{T}_{\text {VRS }}^{\text {HD }}$, and the corresponding misspecification bias $\theta_{o}^{\mathrm{B}}$ calculated by $(9)$.

In conclusion, we state the dual to program (8). Denote $\mathbf{v} \equiv\left(\mathbf{v}_{S}, \mathbf{v}_{W}, \mathbf{v}_{S W}\right)$ the vector whose component subvectors are dual to constraints (8b)-(8d). Let $\mathbf{u} \equiv\left(\mathbf{u}_{S}, \mathbf{u}_{W}, \mathbf{u}_{S W}\right)$ be the vector of components dual to constraints $(8 \mathrm{e})-(8 \mathrm{~g})$. Let scalar $\omega$ be dual to $(8 \mathrm{~h})$, and let vector $\mathbf{z}$ be dual to (8i). Then the dual to (8), or its multiplier form, can be stated as follows:

$$
\begin{array}{ll}
\theta_{o}^{\mathrm{HD}}=\max \quad \mathbf{u}^{T} \mathbf{y}_{o}+\omega & \\
\text { subject to } & \\
\mathbf{v}^{T} \mathbf{x}_{o}=1, & \\
\mathbf{u}^{T} \mathbf{y}_{j}-\mathbf{v}^{T} \mathbf{x}_{j}+\omega+z_{j} \leq 0, & \forall j \in \mathcal{J}, \\
\mathbf{u}_{W}^{T} \mathbf{y}_{j}^{W}+\mathbf{u}_{S W}^{T} \mathbf{y}_{j}^{S W}+z_{j} \geq 0, & \forall j \in \mathcal{J}, \\
\mathbf{v}_{W}^{T} \mathbf{x}_{j}^{W}+\mathbf{v}_{S W}^{T} \mathbf{x}_{j}^{S W} \geq 0, & \forall j \in \mathcal{J}, \\
\mathbf{v}_{S}, \mathbf{v}_{S W}, \mathbf{u}_{S}, \mathbf{u}_{S W}, \mathbf{z} \geq \mathbf{0}, \mathbf{v}_{W}, \mathbf{u}_{W}, \omega \text { sign free. } &
\end{array}
$$

Below, we use the dual program (8) for the evaluation of RTS in technology $\mathcal{T}_{\text {VRS }}^{\text {HD }}$.

\subsection{The output-oriented model}

The output radial efficiency of DMU $\left(\mathbf{x}_{o}, \mathbf{y}_{o}\right)$ in technology $\mathcal{T}_{\text {VRS }}^{\mathrm{HD}}$ is equal to the inverse of the maximal output improvement factor (optimal value) $\eta_{o}^{\mathrm{HD}}$ of the following linear program:

$$
\begin{aligned}
& \eta_{o}^{\mathrm{HD}}=\max \quad \eta \\
& \text { subject to } \\
& \sum_{j \in \mathcal{J}} \lambda_{j} \mathbf{x}_{j}^{S} \leq \mathbf{x}_{o}^{S}, \\
& \sum_{j \in \mathcal{J}}\left(\lambda_{j}+\mu_{j}\right) \mathbf{x}_{j}^{W}=\mathbf{x}_{o}^{W}, \\
& \sum_{j \in \mathcal{J}}\left(\lambda_{j}+\mu_{j}\right) \mathbf{x}_{j}^{S W} \leq \mathbf{x}_{o}^{S W},
\end{aligned}
$$


Table 5: The data set in Example 4.

\begin{tabular}{cccc}
\hline DMU & $x$ & $y_{1}$ & $y_{2}$ \\
\hline$A$ & 1 & 5 & 4 \\
$B$ & 1 & 4 & 9 \\
$C$ & 1 & 1 & 5 \\
$D$ & 1 & 1 & 4.5 \\
\hline
\end{tabular}

$$
\begin{aligned}
& \sum_{j \in \mathcal{J}} \lambda_{j} \mathbf{y}_{j}^{S} \geq \eta \mathbf{y}_{o}^{S}, \\
& \sum_{j \in \mathcal{J}}\left(\lambda_{j}-\nu_{j}\right) \mathbf{y}_{j}^{W}=\eta \mathbf{y}_{o}^{W}, \\
& \sum_{j \in \mathcal{J}}\left(\lambda_{j}-\nu_{j}\right) \mathbf{y}_{j}^{S W} \geq \eta \mathbf{y}_{o}^{S W}, \\
& \mathbf{1}^{T} \boldsymbol{\lambda}=1 \\
& \boldsymbol{\lambda}-\boldsymbol{\nu} \geq \mathbf{0}, \\
& \boldsymbol{\lambda}, \boldsymbol{\mu}, \boldsymbol{\nu} \geq \mathbf{0}, \eta \text { sign free. }
\end{aligned}
$$

It is clear that $\eta_{o}^{\mathrm{HD}} \geq 1 .{ }^{11}$ Program (11) identifies the output radial projection $\left(\mathbf{x}_{o}, \eta_{o}^{\mathrm{HD}} \mathbf{y}_{o}\right)$ of the assessed DMU on the boundary of technology $\mathcal{T}_{\text {VRS }}^{\mathrm{HD}}$.

Let $\eta_{o}^{\mathrm{VRS}}$ be the maximal output improvement factor (the inverse of the output radial efficiency) evaluated in technology $\mathcal{T}_{\text {VRS }}$. As follows from the embedding (4), we always have $\eta_{o}^{\mathrm{HD}} \leq \eta_{o}^{\mathrm{VRS}}$. Similar to the previously considered case, define the ratio

$$
\eta_{o}^{\mathrm{B}}=\frac{\eta_{o}^{\mathrm{HD}}}{\eta_{o}^{\mathrm{VRS}}} .
$$

The ratio $0<\eta_{o}^{\mathrm{B}} \leq 1$ may be viewed as a measure of the misspecification bias arising from the evaluation of the output radial efficiency of DMU $\left(\mathbf{x}_{o}, \mathbf{y}_{o}\right)$ in the conventional VRS technology in the cases in which the inputs and outputs are not all strongly disposable and can be assumed to satisfy only the weaker Axioms SSD and SWD. ${ }^{12}$

The following example illustrates the evaluation of the output radial efficiency in technology $\mathcal{T}_{\text {VRS }}^{\mathrm{HD}}$.

Example 4. Consider the four DMUs $A, B, C$ and $D$ shown in Table 5, whose efficiency is assessed using one input and two outputs. Because the input level of all DMUs is the same, we provide an illustration in the output dimensions only (Fig. 3).

Let output $y_{2}$ be strongly disposable and both outputs $y_{1}$ and $y_{2}$ be jointly weakly disposable. Formally, we define $\mathcal{O}^{S}=\varnothing, \mathcal{O}^{W}=\left\{y_{1}\right\}$ and $\mathcal{O}^{S W}=\left\{y_{2}\right\}$. The corresponding technology $\mathcal{T}_{\mathrm{VRS}}^{\mathrm{HD}}$ is shown (in the output dimensions) as the shaded area $O C B A A^{\prime}$. For comparison purposes, the VRS technology is the larger area $O B^{\prime} B A A^{\prime}$.

\footnotetext{
${ }^{11}$ Indeed, because DMU $\left(\mathbf{x}_{o}, \mathbf{y}_{o}\right) \in \mathcal{T}_{\mathrm{VRS}}^{\mathrm{HD}}$, it satisfies $(7)$ with some vectors $\hat{\boldsymbol{\lambda}}, \hat{\boldsymbol{\mu}}$ and $\hat{\boldsymbol{\nu}}$. These vectors and $\eta=1$ are a feasible solution of program (11). Therefore, $\eta_{o}^{\mathrm{HD}} \geq 1$.

${ }^{12}$ Let all inputs be weakly disposable $\left(\mathcal{I}^{W}=\mathcal{I}\right)$ and all outputs be strongly disposable $\left(\mathcal{O}^{S}=\mathcal{O}\right)$. As shown by Färe and Grosskopf (1983), in this case the ratio $\eta_{o}^{\mathrm{B}}$ in (12) is interpretable as a measure of the input congestion.
} 


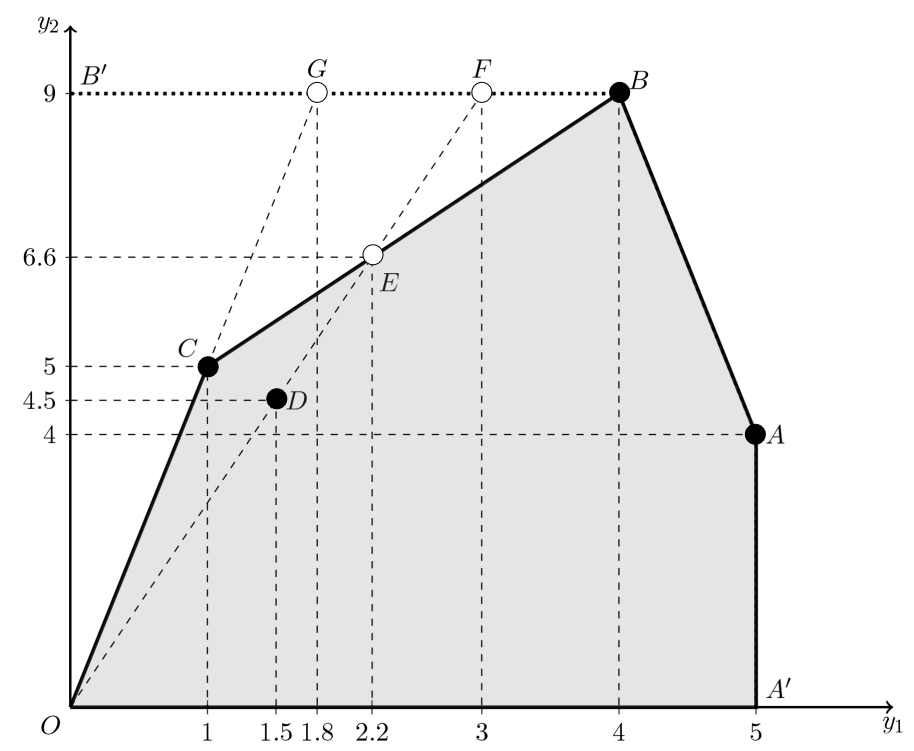

Figure 3: Technology $\mathcal{T}_{\text {VRS }}^{\mathrm{HD}}$ in Example 4.

Table 6: Output radial efficiency and misspecification bias in Example 4.

\begin{tabular}{cccc}
\hline DMU & $1 / \eta_{o}^{\mathrm{VRS}}$ & $1 / \eta_{o}^{\mathrm{HD}}$ & $\eta_{o}^{\mathrm{B}}$ \\
\hline$A$ & 1 & 1 & 1 \\
$B$ & 1 & 1 & 1 \\
$C$ & 0.556 & 1 & 0.556 \\
$D$ & 0.5 & 0.682 & 0.733 \\
\hline
\end{tabular}

As an illustration of Theorem 4.6 note that both technologies have the same strongly efficient frontier represented by the segment $A B$. The weak efficient frontier of the HD technology (for the fixed level of input $x=1$ ) is $A^{\prime} A B$, which is a proper subset of the weak efficient frontier $A^{\prime} A B B^{\prime}$ of the VRS technology.

As in Färe et al. (1985), define the Farrell frontier as the set of DMUs whose technical (in this example, output radial) efficiency is equal to 1 . The Farrell frontier of the VRS technology is its weak efficient frontier $A^{\prime} A B B^{\prime}$. The Farrell frontier of the HD technology is the union of three segments $A^{\prime} A B C$. Note that neither frontier is a subset of the other.

Observe that DMU $C$ is output radial efficient in technology $\mathcal{T}_{\text {VRS }}^{\text {HD }}$ but is inefficient in

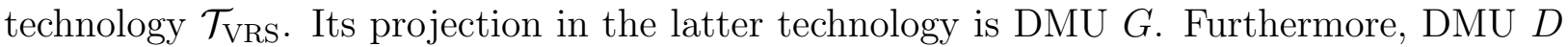
is output radial inefficient in both technologies but its projection $E$ in technology $\mathcal{T}_{\mathrm{VRS}}^{\mathrm{HD}}$ is less demanding in terms of the output levels than its projection $F$ in technology $\mathcal{T}_{\text {VRs }}$.

Table 6 shows the output radial efficiency of the observed DMUs in technologies $\mathcal{T}_{\text {VRS }}$ and $\mathcal{T}_{\mathrm{VRS}}^{\mathrm{HD}}$, and the corresponding misspecification bias $\eta_{o}^{\mathrm{B}}$ calculated by (12).

Remark 1. Examples 3 and 4 may suggest that the incorrect specification of the strong disposability of inputs or outputs, instead of their weak disposability, may lead to a misspecification bias only in the input-oriented or output-oriented models, respectively. This is, however, not so, and the misspecification bias may be observed in both orientations, regardless of whether the inputs or outputs are incorrectly assumed to be strongly disposable.

For example, consider technology $\mathcal{T}_{\text {VRS }}^{\text {HD }}$ in Fig. 1. In this technology, DMU $P$ (located on 


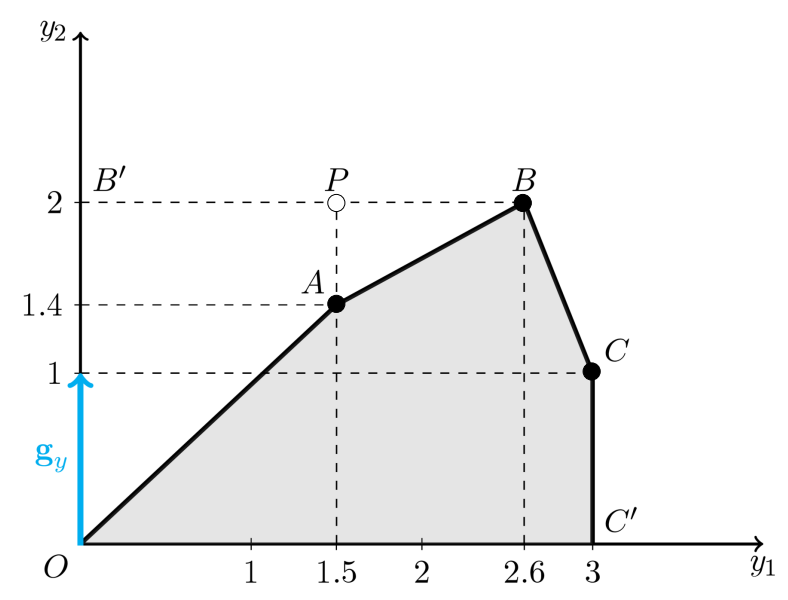

Figure 4: Using a non-radial efficiency measure in Example 5.

the ray $A E$ ) is output radial efficient. However, if we use technology $\mathcal{T}_{\text {VRS }}$, DMU $P$ becomes output radial inefficient, and its output radial projection is point $V$. In this example, although we assume the weak disposability of the inputs, the incorrect use of technology $\mathcal{T}_{\text {VRS }}$ results in the misspecification bias in the output orientation.

Using similar notation $\mathbf{v} \equiv\left(\mathbf{v}_{S}, \mathbf{v}_{W}, \mathbf{v}_{S W}\right), \mathbf{u} \equiv\left(\mathbf{u}_{S}, \mathbf{u}_{W}, \mathbf{u}_{S W}\right), \omega$ and $\mathbf{z}$ for the dual variables as in the previously considered input-oriented case, we state the dual to the outputoriented program (11) as follows:

$$
\begin{array}{ll}
\eta_{o}^{\mathrm{HD}}=\min \quad \mathbf{v}^{T} \mathbf{x}_{o}+\omega & \\
\text { subject to } & \\
\mathbf{u}^{T} \mathbf{y}_{o}=1, & \forall j \in \mathcal{J}, \\
\mathbf{v}^{T} \mathbf{x}_{j}-\mathbf{u}^{T} \mathbf{y}_{j}+\omega-z_{j} \geq 0, & \forall j \in \mathcal{J}, \\
\mathbf{u}_{W}^{T} \mathbf{y}_{j}^{W}+\mathbf{u}_{S W}^{T} \mathbf{y}_{j}^{S W}+z_{j} \geq 0, & \forall j \in \mathcal{J}, \\
\mathbf{v}_{W}^{T} \mathbf{x}_{j}^{W}+\mathbf{v}_{S W}^{T} \mathbf{x}_{j}^{S W} \geq 0, & \\
\mathbf{v}_{S}, \mathbf{v}_{S W}, \mathbf{u}_{S}, \mathbf{u}_{S W}, \mathbf{z} \geq \mathbf{0}, \mathbf{v}_{W}, \mathbf{u}_{W}, \omega \text { sign free. } &
\end{array}
$$

We use the above dual program in the next section for the evaluation of RTS in technology $\mathcal{T}_{\text {VRS }}^{\mathrm{HD}}$.

\subsection{Non-radial models}

Similar to the cases of the input and output radial efficiency measures considered above, it is straightforward to implement non-radial efficiency measures, for example the directional distance function of Chambers, Chung, and Färe (1998), for the assessment of efficiency in technology $\mathcal{T}_{\text {VRS }}^{\mathrm{HD}}$.

The following example utilizes a non-radial efficiency measure. It shows that the use of the VRS technology when the outputs are closely related may lead not only to a misspecification bias, but also to clearly meaningless projections and efficiency scores.

Example 5. Fig. 4 shows technology $\mathcal{T}_{\text {VRS }}^{\text {HD }}$ in two output dimensions $y_{1}$ and $y_{2}$ generated by three observed DMUs $A, B$ and $C$. The inputs of these DMUs are assumed to be identical and are not shown. Suppose that output $y_{2}$ represents a subset of output $y_{1}$. For example, in 
the context of school assessment, let $y_{1}$ be the total number of pupils and $y_{2}$ be the number of pupils who have achieved good academic results.

As argued in Section 1, it should be unrealistic to assume that both outputs are strongly disposable. A more plausible assumption is that both outputs are jointly weakly disposable and that, in addition, output $y_{2}$ is also strongly disposable. Under these assumptions, technology $\mathcal{T}_{\mathrm{VRS}}^{\mathrm{HD}}$ (in the output dimensions) is the shaded area $O A B C C^{\prime}$ in Fig. 5. For reference, the conventional VRS technology is the polyhedron $O B^{\prime} B C C^{\prime}$.

Suppose that the number of all pupils of each school is outside its control and is therefore non-discretionary. Therefore, we may be interested in the evaluation of the efficiency of each school with respect to the discretionary output $y_{2}$ only (Banker \& Morey, 1986). This is equivalent to the use of the directional vectors $\mathbf{g}_{x}=\mathbf{0}$ and $\mathbf{g}_{y}=(0,1)^{T}$ of Chambers et al. (1998).

For the stated non-radial efficiency measure, the efficient frontier is the broken line $O A B C$. Hence, all three observed DMUs $A, B$ and $C$ are efficient. However, if we use the VRS technology, DMU $A$ becomes inefficient and is projected on DMU $P$ on its efficient frontier $B^{\prime} B C$. Note that, at this projection, $y_{1}=1.5$ and $y_{2}=2$, which is impossible because $y_{2}$ represents a subset of $y_{1}$.

\section{Returns to scale in the HD technology}

The notion of returns to scale (RTS) and methods of its evaluation in the VRS technology are well-established in the literature (Førsund \& Hjalmarsson, 2004; Hadjicostas \& Soteriou, 2006; Podinovski \& Førsund, 2010; Sahoo \& Tone, 2015; Zelenyuk, 2013). The HD technology $\mathcal{T}_{\text {VRS }}^{\mathrm{HD}}$ is different from the VRS technology, and the RTS characterization in this case needs discussing.

By Theorem 4.5, technology $\mathcal{T}_{\text {VRS }}^{\text {HD }}$ is a polyhedral set. Podinovski, Chambers, Atici, and Deineko (2016) develop a unifying approach to the definition and evaluation of RTS and other marginal scale characteristics in any polyhedral technology, of which $\mathcal{T}_{\text {VRS }}^{\mathrm{HD}}$ is a special case. Below, we show how these general results apply and can be operationalized in technology $\mathcal{T}_{\mathrm{VRS}}^{\mathrm{HD}}$.

\subsection{Scale elasticity}

In the traditional economic setting assuming a smooth production frontier, the RTS characterization of a DMU $\left(\mathbf{x}_{o}, \mathbf{y}_{o}\right)$ located on the frontier is determined by the scale elasticity $\varepsilon_{o}$ evaluated at this DMU. The scale elasticity $\varepsilon_{o}$ represents the rate of proportional change of the output vector $\mathbf{y}_{o}$ with respect to a marginal proportional change of the input vector $\mathbf{x}_{o}$, assuming the resulting DMU remains on the production frontier. If $\varepsilon_{o}>1, \varepsilon_{o}<1$ or $\varepsilon_{o}=1$, $\operatorname{DMU}\left(\mathbf{x}_{o}, \mathbf{y}_{o}\right)$ is said to exhibit increasing, decreasing and constant RTS, respectively.

If the production frontier is not smooth, like in an arbitrary polyhedral technology, including technologies $\mathcal{T}_{\text {VRS }}$ and $\mathcal{T}_{\text {VRS }}^{\mathrm{HD}}$, the RTS characterization can still be given based on the notions of the one-sided (left-hand and right-hand) scale elasticities $\varepsilon_{o}^{-}$and $\varepsilon_{o}^{+}$, respectively. The former applies if the input vector $\mathbf{x}_{o}$ is reduced in a marginal proportion, and the latter applies if the vector $\mathbf{x}_{o}$ is increased in a marginal proportion. Let us define these notions formally.

Let $\operatorname{DMU}\left(\mathbf{x}_{o}, \mathbf{y}_{o}\right)$ be output radial efficient in technology $\mathcal{T}_{\text {VRS }}^{\mathrm{HD}}$, i.e., $\eta_{o}^{\mathrm{HD}}=1 .{ }^{13}$ To calculate the one-sided scale elasticities $\varepsilon_{o}^{+}$and $\varepsilon_{o}^{-}$in technology $\mathcal{T}_{\mathrm{VRS}}^{\mathrm{HD}}$, we utilize the dual

\footnotetext{
${ }^{13}$ Note that we do not require that the DMU $\left(\mathbf{x}_{o}, \mathbf{y}_{o}\right)$ be strongly efficient, which is sometimes assumed in the literature.
} 
statement of the output-oriented program (13). This is a special case of the general approach applicable to any polyhedral technology considered in Podinovski et al. (2016).

Let $\Omega^{*}$ be the set of optimal solutions of the dual program (13). Because DMU $\left(\mathbf{x}_{o}, \mathbf{y}_{o}\right)$ is output radial efficient, the set $\Omega^{*}$ is defined by conditions (13b)-(13f) and the additional equality requiring that the objective function of program (13) be equal to 1 .

Let $\omega_{\min }$ and $\omega_{\max }$ be, respectively, the minimum and maximum values of the dual variable $\omega$ taken over the set of dual optimal solutions $\Omega^{*}$. Identifying $\omega_{\min }$ and $\omega_{\max }$ requires solving the following two linear programs:

$$
\begin{aligned}
& \omega_{\min }=\min \omega \\
& \text { subject to } \mathbf{v}^{T} \mathbf{x}_{o}+\omega=1,(13 \mathrm{~b})-(13 \mathrm{f}),
\end{aligned}
$$

and

$$
\begin{aligned}
& \omega_{\max }=\max \omega \\
& \text { subject to } \mathbf{v}^{T} \mathbf{x}_{o}+\omega=1,(13 \mathrm{~b})-(13 \mathrm{f}) .
\end{aligned}
$$

According to the general formula for any polyhedral technology established by Podinovski et al. (2016), the one-sided scale elasticities $\varepsilon_{o}^{+}$and $\varepsilon_{o}^{-}$in technology $\mathcal{T}_{\mathrm{VRS}}^{\mathrm{HD}}$ are calculated as follows:

$$
\varepsilon_{o}^{+}=1-\omega_{\max }, \quad \varepsilon_{o}^{-}=1-\omega_{\min } .
$$

As follows from program (15), we always have $\omega_{\max } \leq 1$. $^{14}$ Therefore, $\varepsilon_{o}^{+}=1-\omega_{\max } \geq 0$. At the same time, the objective function of program (14) may be unbounded. As shown in Podinovski et al. (2016), this case arises if and only if it is not possible to reduce the input vector of the DMU $\left(\mathbf{x}_{o}, \mathbf{y}_{o}\right)$ in any marginal proportion because doing so would lead outside the technology. In this case we formally take $\omega_{\min }=-\infty$ and, by $(16), \varepsilon_{o}^{-}=+\infty$.

Equalities (16) and the fact that $\varepsilon_{o}^{+} \geq 0$ established above imply the following relationship: ${ }^{15}$

$$
0 \leq \varepsilon_{o}^{+} \leq \varepsilon_{o}^{-}
$$

Remark 2. If DMU $\left(\mathbf{x}_{o}, \mathbf{y}_{o}\right)$ is both input and output radial efficient, i.e., the optimal values of both programs (8) and (11) are equal to 1, the one-sided scale elasticities $\varepsilon_{o}^{+}$and $\varepsilon_{o}^{-}$can also be obtained using the set $\hat{\Omega}$ of optimal solutions to the dual input-oriented program (10). Let $\hat{\omega}_{\min }$ and $\hat{\omega}_{\max }$ be, respectively, the minimum and maximum values of the dual variable $\omega$ taken over the set $\hat{\Omega}$. (These values are assessed by solving two linear programs (14) and (15) whose constraints are changed to $\mathbf{u}^{T} \mathbf{y}_{o}+\omega=1$ and (10b)-(10f).) Then, as follows from Podinovski et al. (2016),

$$
\varepsilon_{o}^{+}=1 /\left(1-\hat{\omega}_{\max }\right), \quad \varepsilon_{o}^{-}=1 /\left(1-\hat{\omega}_{\min }\right) .
$$

\subsection{Returns to scale}

Let DMU $\left(\mathbf{x}_{o}, \mathbf{y}_{o}\right)$ be output radial efficient in technology $\mathcal{T}_{\text {VRS }}^{\mathrm{HD}}$. Following the definition of Banker and Thrall (1992), extended to arbitrary polyhedral technologies by Podinovski et al. (2016), the type of RTS exhibited by DMU $\left(\mathbf{x}_{o}, \mathbf{y}_{o}\right)$ is defined by the one-sided scale elasticities $\varepsilon_{o}^{+}$and $\varepsilon_{o}^{-}$evaluated at this DMU as follows:

\footnotetext{
${ }^{14}$ For any feasible solution of program (15), we have $\mathbf{v}^{T} \mathbf{x}_{o}+\omega=1$. Because $\mathbf{v}^{T} \mathbf{x}_{o} \geq 0$, we have $\omega \leq 1$.

${ }^{15}$ Podinovski (2017) proves that the inequality $\varepsilon_{o}^{+} \leq \varepsilon_{o}^{-}$is true in any convex technology. However, the nonnegativity of the one-sided scale elasticities stated in (17) is generally not true in an arbitrary convex or, more narrowly, polyhedral technology. The fact that the inequality (17) is true in technology $\mathcal{T}_{\text {VRS }}^{\mathrm{HD}}$ is important for a meaningful characterization of returns to scale in this technology.
} 
Definition 6.1. The output radial efficient DMU $\left(\mathbf{x}_{o}, \mathbf{y}_{o}\right) \in \mathcal{T}_{\mathrm{VRS}}^{\mathrm{HD}}$ exhibits:

(a) increasing returns to scale (IRS) if $1<\varepsilon_{o}^{+} \leq \varepsilon_{o}^{-}$.

(b) decreasing returns to scale (DRS) if $\varepsilon_{o}^{+} \leq \varepsilon_{o}^{-}<1$.

(c) constant returns to scale (CRS) if $\varepsilon_{o}^{+} \leq 1 \leq \varepsilon_{o}^{-}$.

Taking into account (16), Definition 6.1 can be restated directly in terms of the values $\omega_{\min }$ and $\omega_{\max }$. Namely, the DMU $\left(\mathbf{x}_{o}, \mathbf{y}_{o}\right)$ exhibits IRS if $\omega_{\min } \leq \omega_{\max }<0$, DRS if $0<$ $\omega_{\min } \leq \omega_{\max }$, and CRS if $\omega_{\min } \leq 0 \leq \omega_{\max }$.

It is worth noting that the above characterization of RTS is based on the notions of one-sided scale elasticities $\varepsilon_{o}^{+}$and $\varepsilon_{o}^{-}$which are correctly defined only on the production frontier, more precisely, at all output radial efficient DMUs in technology $\mathcal{T}_{\text {VRS }}^{\mathrm{HD}}$. In the DEA literature, the RTS characterization is also sometimes extended to the inefficient DMUs whose type of RTS is defined at its output or input radial projection on the boundary of the technology. ${ }^{16}$ It is well-known that this approach may lead to different characterizations of RTS obtained in the input and output orientations (Banker, Seiford, Cooper, \& Zhu, 2011).

Remark 3. The three types of RTS provide qualitative indicators of the rate of response of the output vector with respect to marginal proportional changes of the input vector. For example, in the case of IRS, an increase of the input vector in a marginal proportion would result in a larger proportional increase of the output vector. The scale elasticities $\varepsilon_{o}^{+}$and $\varepsilon_{o}^{-}$can be seen as the measures that quantify the strength of the RTS types.

Let $\operatorname{DMU}\left(\mathbf{x}_{o}, \mathbf{y}_{o}\right)$ be output radial efficient, i.e., in program (13), we have $\eta_{o}^{\mathrm{HD}}=1$. If we are not interested in the one-sided scale elasticities and require only the RTS characterization of DMUs, this task can be accomplished by solving the following single linear program: ${ }^{17}$

$$
\begin{aligned}
& \omega^{*}=\max \omega \\
& \text { subject to } \\
& \omega \leq 0, \\
& \mathbf{v}^{T} \mathbf{x}_{o}+\omega=1,(13 \mathrm{~b})-(13 \mathrm{f}) .
\end{aligned}
$$

If program (18) is infeasible, DMU $\left(\mathbf{x}_{o}, \mathbf{y}_{o}\right)$ exhibits DRS. Otherwise, the optimal value of program (18) is finite and two cases arise: $\omega^{*}<0$ or $\omega^{*}=0$, which correspond to the cases of IRS and CRS, respectively. A symmetrical approach can be used by replacing the maximization of the objective function in (18) by its minimization, in which case the constraint $\omega \leq 0$ is replaced by $\omega \geq 0$.

Remark 4. According to the definition of Banker (1984), a DMU $\left(\mathbf{x}_{o}, \mathbf{y}_{o}\right)$ is at the most productive scale size (MPSS) in technology $\mathcal{T}$ if, for all DMUs $\left(\alpha \mathbf{x}_{o}, \beta \mathbf{y}_{o}\right) \in \mathcal{T}$, we have $\beta / \alpha \leq 1$.

In the case of VRS technology, Banker and Thrall (1992) prove that DMU $\left(\mathbf{x}_{o}, \mathbf{y}_{o}\right)$ is at MPSS if and only if it exhibits CRS. For an arbitrary convex technology, the equivalence of MPSS and the CRS type is established by Podinovski (2017). In particular, this general result implies that a DMU $\left(\mathbf{x}_{o}, \mathbf{y}_{o}\right) \in \mathcal{T}_{\mathrm{VRS}}^{\mathrm{HD}}$ is at MPSS if and only if it exhibits CRS according to Definition 6.1.

\footnotetext{
${ }^{16}$ Defining the RTS type of an inefficient DMU at its projection still requires that the projection be output radial efficient. This requirement is obviously always true at the output radial projection but may not be true at the input radial projection.

${ }^{17} \mathrm{~A}$ similar approach is obviously applicable in the case of the conventional VRS technology.
} 
Remark 5. In some applications, we may regard only some inputs and outputs as discretionary, while considering the remaining inputs and outputs as non-discretionary. Examples include environmental measures or inputs and outputs set at a higher level of management outside the control of individual DMUs. Furthermore, in considering short run production, certain inputs such as assets and land may also be regarded as non-discretionary measures. ${ }^{18}$

In the described scenario, we may be interested in a partial RTS characterization of DMUs with respect to the subvectors of discretionary inputs and outputs only, while keeping the non-discretionary inputs and outputs constant. Podinovski et al. (2016) develop a linear programming approach for this purpose which utilizes the notion of partial onesided scale elasticities evaluated with respect to the selected subsets of inputs and outputs. This approach is also suitable for the calculation of various marginal rates, such as the rates of transformation and substitution. The described approach applies to any polyhedral technology, including $\mathcal{T}_{\text {VRS }}^{\mathrm{HD}}$.

\section{The extended HD technology}

Below we extend our results to the case in which we have several independent groups of weakly disposable inputs or outputs, numbered by $g=1, \ldots, k$ and $h=1, \ldots, l$, respectively. Because this extension is similar to the previously considered case of technology $\mathcal{T}$ VRS , we briefly outline only the main results.

Consider the following decomposition of the sets of all inputs and outputs into the mutually disjoint subsets:

$$
\mathcal{I}=\mathcal{I}^{S} \cup\left(\bigcup_{g=1}^{k}\left(\mathcal{I}_{g}^{W} \cup \mathcal{I}_{g}^{S W}\right)\right), \quad \mathcal{O}=\mathcal{O}^{S} \cup\left(\bigcup_{h=1}^{l}\left(\mathcal{O}_{h}^{W} \cup \mathcal{O}_{h}^{S W}\right)\right)
$$

Then each DMU $(\mathbf{x}, \mathbf{y})$ can be stated as follows:

$$
(\mathbf{x}, \mathbf{y}) \equiv\left(\mathbf{x}^{S}, \mathbf{x}_{1}^{W}, \mathbf{x}_{1}^{S W}, \ldots, \mathbf{x}_{k}^{W}, \mathbf{x}_{k}^{S W}, \mathbf{y}^{S}, \mathbf{y}_{1}^{W}, \mathbf{y}_{1}^{S W}, \ldots, \mathbf{y}_{l}^{W}, \mathbf{y}_{l}^{S W}\right)
$$

where each individual subvector corresponds to a particular subset in (19).

We assume that the inputs from the group $\mathbf{x}^{S}$ are strongly disposable. For each $g=$ $1, \ldots, k$, the inputs from the union $\mathcal{I}_{g}^{W} \cup \mathcal{I}_{g}^{S W}$ are jointly weakly disposable. Additionally, the inputs in the group $\mathcal{I}_{g}^{S W}$ are also strongly disposable. A similar assumption is made about the outputs.

The following two axioms generalize Axioms SSD and SWD, and describe the above assumptions formally.

Axiom ESSD (Extended Selective Strong Disposability) If $(\mathbf{x}, \mathbf{y}) \in \mathcal{T}$, then

$$
\left(\tilde{\mathbf{x}}^{S}, \mathbf{x}_{1}^{W}, \tilde{\mathbf{x}}_{1}^{S W}, \ldots, \mathbf{x}_{k}^{W}, \tilde{\mathbf{x}}_{k}^{S W}, \tilde{\mathbf{y}}^{S}, \mathbf{y}_{1}^{W}, \tilde{\mathbf{y}}_{1}^{S W}, \ldots, \mathbf{y}_{l}^{W}, \tilde{\mathbf{y}}_{l}^{S W}\right) \in \mathcal{T}
$$

for all $\tilde{\mathbf{x}}^{S} \geq \mathbf{x}^{S}, \tilde{\mathbf{x}}_{g}^{S W} \geq \mathbf{x}_{g}^{S W}(g=1, \ldots, k), \mathbf{0} \leq \tilde{\mathbf{y}}^{S} \leq \mathbf{y}^{S}$ and $\mathbf{0} \leq \tilde{\mathbf{y}}_{h}^{S W} \leq \mathbf{y}_{h}^{S W}(h=1, \ldots, l)$.

The second axiom allows independent weak disposability of different groups of inputs and outputs by specifying different scaling factors attached to them.

Axiom ESWD (Extended Selective Weak Disposability) If $(\mathbf{x}, \mathbf{y}) \in \mathcal{T}$, then

$$
\left(\mathbf{x}^{S}, \rho_{1} \mathbf{x}_{1}^{W}, \rho_{1} \mathbf{x}_{1}^{S W}, \ldots, \rho_{k} \mathbf{x}_{k}^{W}, \rho_{k} \mathbf{x}_{k}^{S W}, \mathbf{y}^{S}, \sigma_{1} \mathbf{y}_{1}^{W}, \sigma_{1} \mathbf{y}_{1}^{S W}, \ldots, \sigma_{l} \mathbf{y}_{l}^{W}, \sigma_{l} \mathbf{y}_{l}^{S W}\right) \in \mathcal{T}
$$

\footnotetext{
${ }^{18}$ Banker and Morey (1986) develop models for the assessment of efficiency and scale efficiency of DMUs in the VRS technology in which a subset of inputs is discretionary.
} 
for all $\rho_{g} \geq 1(g=1, \ldots, k)$ and $0 \leq \sigma_{h} \leq 1(h=1, \ldots, l)$.

In line with the minimum extrapolation principle, we define the extended closed convex HD technology as follows.

Definition 7.1. Technology $\mathcal{T}_{\text {VRS }}^{\text {EHD }}$ is the intersection of all technologies $\mathcal{T} \subset \mathbb{R}_{+}^{m} \times \mathbb{R}_{+}^{s}$ that satisfy Axioms IO, CT, ESSD, ESWD and C.

It is straightforward to prove that technology $\mathcal{T}_{\text {VRS }}^{\mathrm{EHD}}$ itself satisfies the Axioms IO, CT, ESSD, ESWD and C. Therefore, $\mathcal{T}_{\text {VRS }}^{\mathrm{EHD}}$ is the smallest technology that satisfies them.

Similar to our main development of technology $\mathcal{T}_{\text {VRS }}^{\text {HD }}$, we have the following statement of technology $\mathcal{T}_{\text {VRS }}^{\mathrm{EHD}}$, which is a generalization of Theorem 4.4.

Theorem 7.1. Technology $\mathcal{T}$ VRS $\mathrm{EHD}$ stated as follows:

$$
\begin{aligned}
\mathcal{T}_{\mathrm{VRS}}^{\mathrm{EHD}}=\left\{(\mathbf{x}, \mathbf{y}) \in \mathbb{R}_{+}^{m} \times \mathbb{R}_{+}^{s} \mid\right. & \exists \boldsymbol{\lambda} \in \mathbb{R}^{n}, \boldsymbol{\mu}^{g} \in \mathbb{R}^{n}, g=1, \ldots, k, \boldsymbol{\nu}^{h} \in \mathbb{R}^{n}, h=1, \ldots, l: \\
& \sum_{j \in \mathcal{J}} \lambda_{j} \mathbf{x}_{j}^{S} \leq \mathbf{x}^{S}, \\
& \sum_{j \in \mathcal{J}}\left(\lambda_{j}+\mu_{j}^{g}\right) \mathbf{x}_{j, g}^{W}=\mathbf{x}_{g}^{W}, \quad g=1, \ldots, k, \\
& \sum_{j \in \mathcal{J}}\left(\lambda_{j}+\mu_{j}^{g}\right) \mathbf{x}_{j, g}^{S W} \leq \mathbf{x}_{g}^{S W}, \quad g=1, \ldots, k, \\
& \sum_{j \in \mathcal{J}} \lambda_{j} \mathbf{y}_{j}^{S} \geq \mathbf{y}^{S}, \\
& \sum_{j \in \mathcal{J}}\left(\lambda_{j}-\nu_{j}^{h}\right) \mathbf{y}_{j, h}^{W}=\mathbf{y}_{h}^{W}, \quad h=1, \ldots, l, \\
& \sum_{j \in \mathcal{J}}\left(\lambda_{j}-\nu_{j}^{h}\right) \mathbf{y}_{j, h}^{S W} \geq \mathbf{y}_{h}^{S W}, \quad h=1, \ldots, l, \\
& \left.\mathbf{1}^{T} \boldsymbol{\lambda}=1, \boldsymbol{\lambda} \geq \boldsymbol{\nu}^{h}, \forall h, \boldsymbol{\lambda}, \boldsymbol{\mu}^{g}, \boldsymbol{\nu}^{h} \geq \mathbf{0}, \forall g, h\right\} .
\end{aligned}
$$

The proof of Theorem 7.1 is similar to the proof of Theorem 4.4, and is omitted.

In the above statement, $\mathbf{x}_{j, g}^{W}, \mathbf{x}_{j, g}^{S W}, \mathbf{y}_{j, h}^{W}$ and $\mathbf{y}_{j, h}^{S W}$ are the subvectors of the observed DMU $\left(\mathbf{x}_{j}, \mathbf{y}_{j}\right)$ corresponding to the groups of inputs $\mathcal{I}_{g}^{W}$ and $\mathcal{I}_{g}^{S W}$, and the groups of outputs $\mathcal{O}_{h}^{W}$ and $\mathcal{O}_{h}^{S W}$, respectively. Note that the variable multipliers $\mu_{j}^{g}$ and $\nu_{j}^{h}$ are specific to different groups $g$ and $h$ of inputs and outputs. This allows the inputs and outputs in these groups to be weakly disposable independent of the other groups.

Similar to the case of technology $\mathcal{T}_{\text {VRS }}^{\text {HD }}$ which was considered in detail, it is straightforward to state linear programs for the assessment of the input and output radial efficiency of a $\operatorname{DMU}\left(\mathbf{x}_{o}, \mathbf{y}_{o}\right)$ in technology $\mathcal{T}_{\mathrm{VRS}}^{\mathrm{EHD}}$.

Because $\mathcal{T}_{\text {VRS }}^{\mathrm{EHD}}$ is a polyhedral technology, the evaluation of the one-sided scale elasticities $\varepsilon_{o}^{+}$and $\varepsilon_{o}^{-}$at an output radial efficient DMU $\left(\mathbf{x}_{o}, \mathbf{y}_{o}\right)$ and its RTS characterization are a special case of the more general and unifying approach developed by Podinovski et al. (2016). Namely, consider the dual to the linear program that evaluates the output radial efficiency of DMU $\left(\mathbf{x}_{o}, \mathbf{y}_{o}\right)$ in technology $\mathcal{T}_{\mathrm{VRS}}^{\mathrm{EHD}}$. Let $\omega_{\min }$ and $\omega_{\max }$ be, respectively, the minimum and maximum values of the variable $\omega$ dual to the equality $\mathbf{1}^{T} \boldsymbol{\lambda}=1$ in this program. Then the one-sided scale elasticities $\varepsilon_{o}^{+}$and $\varepsilon_{o}^{-}$at DMU $\left(\mathbf{x}_{o}, \mathbf{y}_{o}\right)$ are calculated by formulae (16). The RTS characterization is obtained in line with Definition 6.1. 


\section{Computational experiments with simulated data}

Examples 3 and 4 illustrate the conceptual effect of replacing the assumption of strong disposability by weak disposability on the input and output radial efficiency of DMUs. In this section we provide computational experiments with simulated data that show how noticeable this theoretical effect may be in a typical practical application.

\subsection{The data generating process}

To put the data generating process (DGP) in a practical context, we consider a simple setting arising in the assessment of school efficiency. ${ }^{19}$ We assume that there are two inputs: the number of teachers $x_{1}$, and the overall school expenditure $x_{2}$ (in some monetary units). We also assume that there are two outputs: the number of all pupils $y_{1}$, and the number of pupils with special needs $y_{2}$.

We assume that the theoretical production frontier is defined by the known transformation function as follows: ${ }^{20}$

$$
F\left(x_{1}, x_{2}, y_{1}, y_{2}\right)=y_{1}+0.5 y_{2}-20 x_{1}^{0.8} x_{2}^{0.2}=0 .
$$

Note that not all combinations $\left(x_{1}, x_{2}, y_{1}, y_{2}\right)$ that satisfy $(21)$ can be regarded as valid DMUs. Because pupils with special needs are also included in the number of all pupils, we necessarily have $y_{1} \geq y_{2}$. Furthermore, the DGP employed in this study assumes that the school expenditure is closely related to the number of teachers and allows $x_{2}$ to vary only within certain bounds corresponding to each level of $x_{1}$.

The last term in (21) may be seen as a multiplicative aggregate measure of the overall resource available to the school in which the number of teachers has a higher weight 0.8 compared to the weight 0.2 attached to the expenditure. The transformation function (21) assumes constant rate of transformation between the two outputs. Note that each pupil with special needs is counted twice: in the overall number of pupils $y_{1}$ with the coefficient 1 , and in $y_{2}$ with the coefficient 0.5 . Therefore, we assume that teaching one pupil with special needs requires 1.5 times more resources compared to a pupil without special needs.

A detailed description of the DGP employed in this study is given in Appendix B. According to this DGP, a sample of schools is generated as follows. First, we randomly generate a provisional efficient school $\left(\tilde{x}_{1}, \tilde{x}_{2}, \tilde{y}_{1}, \tilde{y}_{2}\right)$ whose inputs and outputs satisfy equality (21), subject to the restrictions on the inputs and outputs discussed above. We subsequently introduce random inefficiency to the school's outputs. The resulting school is added to the sample.

\subsection{Model specification}

The specification of technology $\mathcal{T}_{\text {VRS }}^{\text {HD }}$ is aligned with our discussion in Section 1. We assume that the number of teachers and school expenditure are closely related inputs, which is modelled by their joint weak disposability. Additionally, we assume that the school expenditure is strongly disposable.

On the output side, we regard all pupils as a measure of the quantity of the services provided by the school, and regard pupils with special needs as a measure of social inclusion. Because pupils with special needs are a subset of all pupils, it is appropriate to treat both

\footnotetext{
${ }^{19}$ Conceptual and practical complexities of the efficiency assessment in an educational context are well known but are not important for the described computational experiment.

${ }^{20} \mathrm{In}$ economics, a transformation function is often used as an implicit definition of the production frontier (Førsund, 1996).
} 
Table 7: Descriptive statistics for the data set in the computational example.

\begin{tabular}{cccccc}
\hline Measure & Mean & Median & Minimum & Maximum & Standard deviation \\
\hline$x_{1}$ & 67 & 66 & 30 & 99 & 19.8 \\
$x_{2}$ & 1161.4 & 1059.5 & 448 & 2073 & 416.3 \\
$y_{1}$ & 434.7 & 390 & 202.3 & 846.7 & 164.7 \\
$y_{2}$ & 79.9 & 79 & 0 & 239.4 & 47.9 \\
\hline
\end{tabular}

Table 8: Summary statistics for the output radial efficiency in different models.

\begin{tabular}{llll}
\hline Model & $\begin{array}{l}\text { Number of } \\
\text { efficient schools }\end{array}$ & $\begin{array}{l}\text { Mean } \\
\text { efficiency }\end{array}$ & $\begin{array}{l}\text { Standard } \\
\text { deviation }\end{array}$ \\
\hline $\mathbb{M}_{\text {VRS }}$ & 12 & 0.8285 & 0.1399 \\
$\mathbb{M}_{\text {HD }}$ & 16 & 0.8392 & 0.1452 \\
$\mathbb{M}_{\mathrm{W}}$ & 25 & 0.8934 & 0.1323 \\
\hline
\end{tabular}

outputs as jointly weakly disposable. We additionally assume strong disposability of pupils with special needs.

Using notation (2), we define technology $\mathcal{T}_{\text {VRS }}^{\mathrm{HD}}$ by the following sets: $\mathcal{I}^{W}=\left\{x_{1}\right\}, \mathcal{I}^{S W}=$ $\left\{x_{2}\right\}, \mathcal{O}^{W}=\left\{y_{1}\right\}$ and $\mathcal{O}^{S W}=\left\{y_{2}\right\}$. We denote $\mathbb{M}_{\text {HD }}$ the output-oriented model (11) based on this technology.

For reference, we also use two further output-oriented models. The first is model $\mathbb{M}_{\mathrm{W}}$ which assumes that both inputs and both outputs are only weakly disposable, and none is strongly disposable, i.e., $\mathcal{I}^{W}=\left\{x_{1}, x_{2}\right\}$ and $\mathcal{O}^{W}=\left\{y_{1}, y_{2}\right\}$. The second is the standard VRS model $\mathbb{M}_{\text {VRS }}$ which assumes strong disposability of all inputs and outputs.

\subsection{Discussion of results}

Taking into account the embedding (4), it is theoretically clear that the model $\mathbb{M}_{\text {VRS }}$ should provide better discrimination on efficiency than both models $\mathbb{M}_{\mathrm{HD}}$ and $\mathbb{M}_{\mathrm{W}}$, and that the latter model, based on the smallest technology, should be the least discriminating of the three models.

In order to assess the extent to which these theoretical differences between the three models could be observed in a typical practical setting, we ran extensive computational experiments with different samples generated by the described DGP. The results of these experiments are consistent. Below we discuss one of them which is based on a sample of 50 schools. Table 7 gives its descriptive statistics.

The computational results obtained from the three models are shown in Table 8. As seen from this table, the number of efficient schools equal to 12 in model $\mathbb{M}_{\text {VRS }}$ increases to 16 in model $\mathbb{M}_{\mathrm{HD}}$, and further increases to 25 if we assume only weak disposability of all inputs and outputs as in model $\mathbb{M}_{\mathrm{W}}$.

In addition to Table 8, our results show that there are 12 schools whose efficiency in model $\mathbb{M}_{\mathrm{HD}}$ is higher than in model $\mathbb{M}_{\mathrm{VRS}}$ (including the four schools that are efficient in the former model but are inefficient in the latter). For these schools, the misspecification bias $\eta_{o}^{\mathrm{B}}$ calculated by formula (12) ranges from the almost negligible 0.9996 to the much more significant 0.7884 , with the mean value of 0.9655 . (This corresponds to the difference 
in efficiency scores changing from 0.0003 to 0.2116 , with the mean value of 0.0332 .) The mean bias $\eta_{o}^{\mathrm{B}}$ calculated for all 50 schools in the sample (including those for which the efficiency in the models $\mathbb{M}_{\mathrm{HD}}$ and $\mathbb{M}_{\mathrm{VRS}}$ is the same) is 0.989 .

Our computations show that, in practical applications, the misspecification bias arising from the incorrect assumption of strong disposability of the inputs and outputs may be significant, and the possibility of obtaining biased efficiency scores (and, consequently, unrealistic projections of the DMUs based on such scores) should not be ignored. In such cases the HD technology $\mathcal{T}_{\text {VRS }}^{\text {HD }}$ may provide a more conservative and arguably safer modelling option.

Table 8 shows that the difference between the mean efficiencies (or, equivalently, the mean difference of efficiencies) between the VRS and HD models is relatively small. This difference may be regarded unimportant if we are interested only in the mean efficiency scores and some other descriptive statistics. In other situations, we may be interested in setting targets for individual inefficient schools for which this discrepancy can be relatively large and, as shown by our example, result in a noticeable misspecification bias. For such schools, the radial projections obtained from the VRS model are unsubstantiated, which justifies the use of the HD model.

In our computations, we also explored the differences in the RTS characterization obtained in models with different disposability assumptions. For the model $\mathbb{M}_{\mathrm{HD}}$, we used programs (14) and (15) to evaluate the two extreme values $\omega_{\min }$ and $\omega_{\max }$, which we further converted to the one-sided scale elasticities $\varepsilon_{o}^{-}$and $\varepsilon_{o}^{+}$by formulae (16). For the model $\mathbb{M}_{\mathrm{VRS}}$, we used a similar approach based on the evaluation of the extreme values $\omega_{\min }$ and $\omega_{\max }$ in the standard multiplier VRS model. In both cases, the computations were performed only for the output radial efficient schools in the respective model. These results were translated to the RTS characterization using Definition 6.1.

Two observations could be made. First, we comment on the 12 schools that are output radial efficient in model $\mathbb{M}_{\text {VRS }}$ (and therefore in model $\mathbb{M}_{\mathrm{HD}}$ ), as shown in Table 8. For 11 of these schools, the RTS characterization is the same in both models $\mathbb{M}_{\mathrm{VRS}}$ and $\mathbb{M}_{\mathrm{HD}}$ (although the strength of this characterization represented by the one-sided scale elasticities is generally different). Out of these 11 schools, one exhibits IRS, 6 exhibit DRS, and 4 exhibit CRS and are therefore at MPSS. The remaining output efficient school exhibits DRS in model $\mathbb{M}_{\text {VRS }}$ and CRS in model $\mathbb{M}_{\text {HD }}$. Second, we consider the remaining 4 schools out of the 16 schools that are output radial efficient in model $\mathbb{M}_{\mathrm{HD}}$ but not in model $\mathbb{M}_{\mathrm{VRS}}$. Applying Definition 6.1 to the former technology only, we establish that three of these DMUs are at MPSS and one exhibits DRS.

\section{Conclusions}

Most models of efficiency analysis are based on the assumption of strong disposability of all inputs and outputs. In this paper we revisit this standard assumption and identify problems that arise if some closely related inputs or outputs are assumed strongly disposable. This includes situations in which an input or output overlaps with another input or, respectively, output, or is a subset of the latter. Another example is a situation in which some inputs or outputs are clearly related or strongly correlated, so that their independent disposal may result in meaningless input or output proportions.

To address these situations, we develop a new production technology in which the groups of closely related input and output measures are assumed to be only jointly weakly disposable. We also assume that some inputs and outputs in these groups may be individually strongly disposable. We formally state these disposability assumptions as production ax- 
ioms. Using these axioms together with the standard axiom of convexity, we define the new hybrid disposable $(\mathrm{HD})$ technology $\mathcal{T}_{\text {VRS }}^{\mathrm{HD}}$. We show that this technology can be stated in the form of linear equalities and inequalities, and that the input and output-oriented models based on it are linear programs.

In an additional development, we introduce the notions of scale elasticity and returns to scale for technology $\mathcal{T}_{\text {VRS }}^{\mathrm{HD}}$, and develop a computational approach for their evaluation. We further generalize technology $\mathcal{T}_{\text {VRS }}^{\mathrm{HD}}$ to the case in which there are several independent groups of weakly disposable inputs and outputs. We show that all the main results obtained for technology $\mathcal{T}_{\text {VRS }}^{\text {HD }}$ remain valid in this extended scenario.

To demonstrate the usefulness of our approach in a practical setting, we provide a computational experiment with a well-defined data generating process. This experiment models a real-life production technology with closely related inputs and outputs in a school assessment context. The two main results can be summarized as follows. First, there is only a relatively minor difference between the mean efficiency scores in the VRS and HD models. The latter model, based on a smaller technology, provides a lower discrimination on efficiency than the former. Second, for some individual DMUs, the discrepancy between the efficiency scores measured by the misspecification bias can be significant. It easy to see that both findings support the usefulness of the HD model in practical applications with closely related inputs and outputs.

Indeed, suppose that we are concerned with the fact that some of the inputs and outputs are (or may be) closely related. The VRS model does not address this close relationship and may produce targets that are technologically impossible. The alternative is to use the HD model. However, this is at the expense of the lower efficiency discrimination, which poses a dilemma. Our experiments show that, in a typical setting, the loss of discrimination is not dramatic, although there is still a difference between the two models. The latter means that, for a minority of DMUs, the VRS model creates unsubstantiated projections (ignoring close relationship), so its results for such DMUs are technologically unexplainable. The former means that the HD model, while removing this concern, still produces acceptable discrimination. Therefore, by using the HD model instead of the VRS model in the described situation, we can increase the reliability (meaningfulness) of the results with just a minor loss of discrimination on efficiency.

An interesting remaining issue is the modelling of the situation in which several groups of weakly disposable inputs share a common input which is additionally strongly disposable. An example of such input is cost which may be incorporated in different groups of weakly disposable inputs. It is clear that individual proportional increase of several groups of inputs with different factors need to be reflected in an acceptable increase of the common input. Modelling this situation is left outside the scope of this paper for future research.

It is also worth considering the incorporation of selective strong and weak disposability assumptions in nonconvex production technologies, including free disposal hull of Deprins, Simar, and Tulkens (1984). Developing the notion of RTS for such technologies presents a further conceptual challenge (Cesaroni, Kerstens, \& Van de Woestyne, 2017). From the theoretical perspective, questions remain open regarding the dual interpretation of the selective disposability assumptions in the sense of Shephard's lemma (Färe, \& Primont, 1995) and, in the context of undesirable outputs, their relationship to the notion of null-jointness (see, e.g., Färe and Grosskopf, 2004). Also worth exploring is the notion of desirable congestion introduced by Sueyoshi and Goto (2012) in relation to the new HD models. Finally, a question of significance tests for the misspecification bias arising from the use of the standard VRS model in the situation in which strong disposability of the closely related inputs and outputs should not be assumed is worth exploring in future research. 


\section{Appendix A. Proofs}

Lemma 1. Denote $\mathcal{K}$ the set on the right-hand side of (3). Then $\mathcal{K}$ satisfies Axioms IO, $C T, S S D$ and $S W D$.

Proof of Lemma 1. Clearly, $\mathcal{K}$ satisfies Axiom IO. To prove that it satisfies Axiom CT, consider any two DMUs $\left(\mathbf{x}^{1}, \mathbf{y}^{1}\right)$ and $\left(\mathbf{x}^{2}, \mathbf{y}^{2}\right)$ from $\mathcal{K}$. These DMUs satisfy (3) with some vectors $\boldsymbol{\lambda}^{1}, \boldsymbol{\rho}^{1}, \boldsymbol{\sigma}^{1}$, and $\boldsymbol{\lambda}^{2}, \boldsymbol{\rho}^{2}, \boldsymbol{\sigma}^{2}$, respectively. For any $\alpha \in[0,1]$, the vector $(\hat{\mathbf{x}}, \hat{\mathbf{y}})=$ $\alpha\left(\mathbf{x}^{1}, \mathbf{y}^{1}\right)+(1-\alpha)\left(\mathbf{x}^{2}, \mathbf{y}^{2}\right)$ satisfies (3) with the vectors $\hat{\boldsymbol{\lambda}}, \hat{\boldsymbol{\rho}}$ and $\hat{\boldsymbol{\sigma}}$, which are defined as follows:

$$
\begin{aligned}
& \hat{\boldsymbol{\lambda}}=\alpha \boldsymbol{\lambda}^{1}+(1-\alpha) \boldsymbol{\lambda}^{2}, \\
& \hat{\rho}_{j}=\left\{\begin{array}{ll}
\frac{1}{\hat{\lambda}_{j}}\left(\alpha \rho_{j}^{1} \lambda_{j}^{1}+(1-\alpha) \rho_{j}^{2} \lambda_{j}^{2}\right), & \text { if } \hat{\lambda}_{j}>0, \\
1, & \text { otherwise, }
\end{array} \in \mathcal{J},\right. \\
& \hat{\sigma}_{j}= \begin{cases}\frac{1}{\hat{\lambda}_{j}}\left(\alpha \sigma_{j}^{1} \lambda_{j}^{1}+(1-\alpha) \sigma_{j}^{2} \lambda_{j}^{2}\right), & \text { if } \hat{\lambda}_{j}>0, \\
1, & \text { otherwise, }\end{cases}
\end{aligned}
$$

Therefore, $(\hat{\mathbf{x}}, \hat{\mathbf{y}}) \in \mathcal{K}$, and $\mathcal{K}$ satisfies Axiom CT. To prove that $\mathcal{K}$ satisfies Axioms SSD and SWD, let $(\mathbf{x}, \mathbf{y}) \in \mathcal{K}$. Then $(\mathbf{x}, \mathbf{y})$ satisfies (3) with some vectors $\overline{\boldsymbol{\lambda}}, \overline{\boldsymbol{\rho}}$ and $\overline{\boldsymbol{\sigma}}$.

The DMU $\left(\tilde{\mathbf{x}}^{S}, \mathbf{x}^{W}, \tilde{\mathbf{x}}^{S W}, \tilde{\mathbf{y}}^{S}, \mathbf{y}^{W}, \tilde{\mathbf{y}}^{S W}\right)$ in the statement of Axiom SSD satisfies (3) with the same vectors $\overline{\boldsymbol{\lambda}}, \overline{\boldsymbol{\rho}}$ and $\overline{\boldsymbol{\sigma}}$ and is in $\mathcal{K}$. Therefore, $\mathcal{K}$ satisfies Axiom SSD.

To prove that $\mathcal{K}$ satisfies Axiom SWD, consider any $\alpha \geq 1$ and $0 \leq \beta \leq 1$. Then $\left(\mathbf{x}^{S}, \alpha \mathbf{x}^{W}, \alpha \mathbf{x}^{S W}, \mathbf{y}^{S}, \beta \mathbf{y}^{W}, \beta \mathbf{y}^{S W}\right)$ satisfies all conditions in (3) with the vectors $\overline{\boldsymbol{\lambda}}, \alpha \overline{\boldsymbol{\rho}}$ and $\beta \overline{\boldsymbol{\sigma}}$, and is therefore in $\mathcal{K}$. This proves that $\mathcal{K}$ satisfies Axiom SWD.

Proof of Theorem 4.1. Let $\mathcal{K}$ be the set on the right-hand side of (3). By Lemma 1, $\mathcal{K}$ satisfies Axioms IO, CT, SSD and SWD. Because $\tilde{\mathcal{T}}$ is the smallest set that satisfies these axioms, we have $\tilde{\mathcal{T}} \subseteq \mathcal{K}$.

Conversely, let $(\mathbf{x}, \mathbf{y}) \in \mathcal{K}$. Then $(\mathbf{x}, \mathbf{y})$ satisfies (3) with some vectors $\overline{\boldsymbol{\lambda}}, \overline{\boldsymbol{\rho}}$ and $\overline{\boldsymbol{\sigma}}$. We need to prove that $(\mathbf{x}, \mathbf{y}) \in \tilde{\mathcal{T}}$. Because $\tilde{\mathcal{T}}$ satisfies Axioms IO and SWD, we have $\left(\mathbf{x}_{j}^{S}, \bar{\rho}_{j} \mathbf{x}_{j}^{W}, \bar{\rho}_{j} \mathbf{x}_{j}^{S W}, \mathbf{y}_{j}^{S}, \bar{\sigma}_{j} \mathbf{y}_{j}^{W}, \bar{\sigma}_{j} \mathbf{y}_{j}^{S W}\right) \in \tilde{\mathcal{T}}$, for all $j \in \mathcal{J}$. Because $\tilde{\mathcal{T}}$ satisfies Axioms CT and $\operatorname{SSD},(\mathbf{x}, \mathbf{y}) \in \tilde{\mathcal{T}}$.

Lemma 2. The following embedding is true: $\tilde{\mathcal{T}} \subseteq \mathcal{T}_{\mathrm{VRS}}^{\mathrm{HD}}$.

Proof of Lemma 2. The set $\mathcal{T}_{\mathrm{VRS}}^{\mathrm{HD}}$ satisfies Axioms IO, CT, SSD and SWD. The proof is completed by noting that $\tilde{\mathcal{T}}$ is the smallest set that satisfies these axioms.

Lemma 3. Denote $\mathcal{P}$ the set on the right-hand side of equality (7). Then $\mathcal{P}$ is a polyhedral set, which implies that it satisfies Axioms $C T$ and $C$. Furthermore, $\mathcal{P}$ satisfies Axioms IO, $S S D$ and $S W D$.

Proof of Lemma 3. The fact that $\mathcal{P}$ is a polyhedral set follows from the projection lemma (see, e.g., the proof of Proposition 1 in Podinovski et al. (2016)). Therefore, $\mathcal{P}$ satisfies Axioms CT and C. Clearly, $\mathcal{P}$ also satisfies Axiom IO. To prove that $\mathcal{P}$ satisfies Axioms SSD and SWD, let $(\mathbf{x}, \mathbf{y}) \in \mathcal{P}$. Then $(\mathbf{x}, \mathbf{y})$ satisfies $(7)$ with some vectors $\overline{\boldsymbol{\lambda}}, \overline{\boldsymbol{\mu}}$ and $\overline{\boldsymbol{\nu}}$.

The DMU $\left(\tilde{\mathbf{x}}^{S}, \mathbf{x}^{W}, \tilde{\mathbf{x}}^{S W}, \tilde{\mathbf{y}}^{S}, \mathbf{y}^{W}, \tilde{\mathbf{y}}^{S W}\right)$ in the statement of Axiom SSD satisfies (7) with the same vectors $\overline{\boldsymbol{\lambda}}, \overline{\boldsymbol{\nu}}$ and $\overline{\boldsymbol{\mu}}$. Therefore, $\mathcal{P}$ satisfies Axiom SSD.

Consider any $\alpha \geq 1$ and $0 \leq \beta \leq 1$. Then $\left(\mathbf{x}^{S}, \alpha \mathbf{x}^{W}, \alpha \mathbf{x}^{S W}, \mathbf{y}^{S}, \beta \mathbf{y}^{W}, \beta \mathbf{y}^{S W}\right)$ satisfies (7) with vectors $\boldsymbol{\lambda}=\overline{\boldsymbol{\lambda}}, \boldsymbol{\mu}=\alpha(\overline{\boldsymbol{\lambda}}+\overline{\boldsymbol{\mu}})-\overline{\boldsymbol{\lambda}}$ and $\boldsymbol{\nu}=\overline{\boldsymbol{\lambda}}-\beta(\overline{\boldsymbol{\lambda}}-\overline{\boldsymbol{\nu}})$. Therefore, $\mathcal{P}$ satisfies Axiom SWD. 
Lemma 4. Let $\mathcal{P}$ be the set on the right-hand side of equality $(7)$. Then $\mathcal{P}=\operatorname{cl}(\tilde{\mathcal{T}})$.

Proof of Lemma 4. If $(\overline{\mathbf{x}}, \overline{\mathbf{y}}) \in \tilde{\mathcal{T}}$, it satisfies (3) with some vectors $\overline{\boldsymbol{\lambda}}, \overline{\boldsymbol{\rho}}$ and $\overline{\boldsymbol{\sigma}}$. Then $(\overline{\mathbf{x}}, \overline{\mathbf{y}})$ satisfies (7) with the same $\overline{\boldsymbol{\lambda}}$ and the vectors $\overline{\boldsymbol{\mu}}$ and $\overline{\boldsymbol{\nu}}$ defined by (5). Therefore $(\overline{\mathbf{x}}, \overline{\mathbf{y}}) \in \mathcal{P}$, and $\tilde{\mathcal{T}} \subseteq \mathcal{P}$. By Lemma $3, \mathcal{P}$ is a closed set. Therefore, $\operatorname{cl}(\tilde{\mathcal{T}}) \subseteq \mathcal{P}$.

Conversely, let $(\overline{\mathbf{x}}, \overline{\mathbf{y}}) \in \mathcal{P}$. Then $(\overline{\mathbf{x}}, \overline{\mathbf{y}})$ satisfies $(7)$ with some vectors $\overline{\boldsymbol{\lambda}}, \overline{\boldsymbol{\mu}}$ and $\overline{\boldsymbol{\nu}}$. We need to prove that $(\overline{\mathbf{x}}, \overline{\mathbf{y}}) \in \operatorname{cl}(\tilde{\mathcal{T}})$. Two cases arise.

Case 1. Suppose that $\overline{\boldsymbol{\lambda}}>\mathbf{0}$. Using (6), define vectors $\overline{\boldsymbol{\rho}}$ and $\overline{\boldsymbol{\sigma}}$ as follows:

$$
\bar{\rho}_{j}=\left(\bar{\lambda}_{j}+\bar{\mu}_{j}\right) / \bar{\lambda}_{j}, \quad \bar{\sigma}_{j}=\left(\bar{\lambda}_{j}-\bar{\nu}_{j}\right) / \bar{\lambda}_{j}, \quad j \in \mathcal{J},
$$

which implies $\bar{\lambda}_{j}+\bar{\mu}_{j}=\bar{\lambda}_{j} \bar{\rho}_{j}$ and $\bar{\lambda}_{j}-\bar{\nu}_{j}=\bar{\lambda}_{j} \bar{\sigma}_{j}$. Note that $\overline{\boldsymbol{\rho}} \geq \mathbf{1}$ and $\mathbf{0} \leq \overline{\boldsymbol{\sigma}} \leq \mathbf{1}$. Then $(\overline{\mathbf{x}}, \overline{\mathbf{y}})$ satisfies $(3)$ with the vectors $\overline{\boldsymbol{\lambda}}, \overline{\boldsymbol{\rho}}$ and $\overline{\boldsymbol{\sigma}}$. Therefore, $(\overline{\mathbf{x}}, \overline{\mathbf{y}}) \in \tilde{\mathcal{T}} \subseteq \operatorname{cl}(\tilde{\mathcal{T}})$.

Case 2. Suppose that the inequality $\overline{\boldsymbol{\lambda}}>\mathbf{0}$ is not true. Let us show that, in this case, $(\overline{\mathbf{x}}, \overline{\mathbf{y}})$ is the limit of some sequence $\left\{\left(\overline{\mathbf{x}}_{t}, \overline{\mathbf{y}}_{t}\right)\right\}, t=1,2 \ldots$, each element of which is in $\tilde{\mathcal{T}}$. Because $\operatorname{cl}(\tilde{\mathcal{T}})$ is a closed set, this would imply that $(\overline{\mathbf{x}}, \overline{\mathbf{y}}) \in \operatorname{cl}(\tilde{\mathcal{T}})$.

To define the required sequence, first let $\left(\mathbf{x}^{*}, \mathbf{y}^{*}\right)$ be the simple average of all observed DMUs $\left(\mathbf{x}_{j}, \mathbf{y}_{j}\right), j \in \mathcal{J}$. Note that $\left(\mathbf{x}^{*}, \mathbf{y}^{*}\right)$ satisfies $(7)$ with vectors $\boldsymbol{\lambda}^{*}=(1 / n) \mathbf{1}$ and $\boldsymbol{\mu}^{*}=\boldsymbol{\nu}^{*}=\mathbf{0}$. Define the elements of the above sequence as follows:

$$
\left(\overline{\mathbf{x}}_{t}, \overline{\mathbf{y}}_{t}\right)=(1-1 / t)(\overline{\mathbf{x}}, \overline{\mathbf{y}})+(1 / t)\left(\mathbf{x}^{*}, \mathbf{y}^{*}\right), \quad t=1,2, \ldots
$$

For any $t=1,2, \ldots$, the DMU $\left(\overline{\mathbf{x}}_{t}, \overline{\mathbf{y}}_{t}\right)$ satisfies (3) with the vectors $\boldsymbol{\lambda}_{t}, \boldsymbol{\mu}_{t}$ and $\boldsymbol{\nu}_{t}$ defined as follows:

$$
\begin{aligned}
& \boldsymbol{\lambda}_{t}=(1-1 / t) \overline{\boldsymbol{\lambda}}+(1 / t) \boldsymbol{\lambda}^{*}, \\
& \boldsymbol{\mu}_{t}=(1-1 / t) \overline{\boldsymbol{\mu}}+(1 / t) \boldsymbol{\mu}^{*}=(1-1 / t) \overline{\boldsymbol{\mu}}, \\
& \boldsymbol{\sigma}_{t}=(1-1 / t) \overline{\boldsymbol{\nu}}+(1 / t) \boldsymbol{\nu}^{*}=(1-1 / t) \overline{\boldsymbol{\nu}} .
\end{aligned}
$$

Because $\boldsymbol{\lambda}_{t}>\mathbf{0}$, as proved in Case $1,\left(\overline{\mathbf{x}}_{t}, \overline{\mathbf{y}}_{t}\right) \in \tilde{\mathcal{T}}$. Then the limit $(\overline{\mathbf{x}}, \overline{\mathbf{y}})$ of this sequence belongs to $\operatorname{cl}(\tilde{\mathcal{T}})$. Therefore, $(\overline{\mathbf{x}}, \overline{\mathbf{y}}) \in \operatorname{cl}(\tilde{\mathcal{T}})$.

The two considered cases imply that $\mathcal{P} \subseteq \operatorname{cl}(\tilde{\mathcal{T}})$.

Proof of Theorem 4.2. Let $\mathcal{I}^{\mathcal{S}}=\mathcal{I}$. Then the two conditions in (3) for vectors $\mathrm{x}^{W}$ and $\mathbf{x}^{S W}$, and the vector $\boldsymbol{\rho}$, are omitted. By substitution $\nu_{j}=\lambda_{j}\left(1-\sigma_{j}\right), \forall j \in \mathcal{J}$, the resulting conditions (3) are equivalently restated as (7) in which the two conditions for vectors $\mathbf{x}^{W}$ and $\mathbf{x}^{S W}$, and the vector $\boldsymbol{\mu}$, are omitted.

To prove equivalence, let (x,y) satisfy (3) with some vectors $\overline{\boldsymbol{\lambda}}$ and $\overline{\boldsymbol{\sigma}}$ (as noted, the vector $\boldsymbol{\rho}$ is omitted). Then $(\mathbf{x}, \mathbf{y})$ satisfies (7) with the same $\overline{\boldsymbol{\lambda}}$ and the vector $\overline{\boldsymbol{\nu}}$ defined by the above substitution. Conversely, let (x,y) satisfy (7) with some vectors $\overline{\boldsymbol{\lambda}}$ and $\overline{\boldsymbol{\nu}}$ (as noted, the vector $\boldsymbol{\mu}$ is omitted). Then (x,y) satisfies (3) with the same $\overline{\boldsymbol{\lambda}}$ and the vector $\overline{\boldsymbol{\sigma}}$ defined as follows: if $\bar{\lambda}_{j}>0$ then $\bar{\sigma}_{j}=\left(\bar{\lambda}_{j}-\bar{\nu}_{j}\right) / \bar{\lambda}_{j}$, and if $\bar{\lambda}_{j}=0$ then arbitrarily $\bar{\sigma}_{j}=1$.

By Lemma 3 , the set $\mathcal{P}$ defined by $(7)$ is a polyhedral set. Therefore, $\tilde{\mathcal{T}}$ is a polyhedral technology.

Proof of Theorem 4.3. By Lemma 3, P satisfies Axioms IO, CT, SSD, SWD and C. By definition of $\mathcal{T}_{\text {VRS }}^{\mathrm{HD}}$, we have $\mathcal{T}_{\text {VRS }}^{\mathrm{HD}} \subseteq \mathcal{P}$. Conversely, by Lemma 2, we have $\tilde{\mathcal{T}} \subseteq \mathcal{T}_{\text {VRS }}^{\mathrm{HD}}$. Then, taking into account Lemma 4 , we have $\mathcal{P}=\operatorname{cl}(\tilde{\mathcal{T}}) \subseteq \operatorname{cl}\left(\mathcal{T}_{\text {VRS }}^{\text {HD }}\right)=\mathcal{T}_{\text {VRS }}^{\text {HD }}$.

Proof of Theorem 4.4. By Theorem 4.3 and Lemma 4, we have $\mathcal{T}_{\text {VRS }}^{\mathrm{HD}}=\operatorname{cl}(\tilde{\mathcal{T}})=\mathcal{P}$, where $\mathcal{P}$ is the set on the right-hand side of equality (7). 
Proof of Theorem 4.5. By Theorem 4.4, $\mathcal{T}_{\text {VRS }}^{\mathrm{HD}}=\mathcal{P}$, where $\mathcal{P}$ is the set on the right-hand side of equality (7). By Lemma $3, \mathcal{P}$ is a polyhedral set.

Proof of Theorem 4.6. Let us prove statement (i). Let DMU $\left(\mathbf{x}^{*}, \mathbf{y}^{*}\right) \in \mathcal{E}_{\mathrm{VRS}}^{*}$. Then it satisfies the input and output inequalities of (1) with some vector $\boldsymbol{\lambda}^{*}$ without slacks, i.e., each input and output inequality is satisfied as equality. Then $\left(\mathbf{x}^{*}, \mathbf{y}^{*}\right)$ satisfies (7) with $\boldsymbol{\lambda}=\boldsymbol{\lambda}^{*}$ and $\boldsymbol{\mu}=\boldsymbol{\nu}=\mathbf{0}$. Therefore $\left(\mathrm{x}^{*}, \mathbf{y}^{*}\right) \in \mathcal{T}_{\mathrm{VRS}}^{\mathrm{HD}}$. Because $\left(\mathbf{x}^{*}, \mathbf{y}^{*}\right)$ is strongly efficient (nondominated) in $\mathcal{T}_{\text {VRS }}$, it is not dominated in its subset $\mathcal{T}_{\text {VRS }}^{\text {HD }}$. Therefore, $\left(\mathbf{x}^{*}, \mathbf{y}^{*}\right) \in \mathcal{E}_{\mathrm{HD}}^{*}$.

Conversely, let DMU $\left(\mathbf{x}^{*}, \mathbf{y}^{*}\right) \in \mathcal{E}_{\mathrm{HD}}^{*}$. By $(4),\left(\mathbf{x}^{*}, \mathbf{y}^{*}\right) \in \mathcal{T}_{\text {VRS }}$. By contradiction, assume that $\left(\mathbf{x}^{*}, \mathbf{y}^{*}\right) \notin \mathcal{E}_{\mathrm{VRS}}^{*}$. Then there exists a DMU $\left(\mathbf{x}^{\prime}, \mathbf{y}^{\prime}\right) \in \mathcal{T}_{\text {VRS }}$, such that $\mathbf{x}^{\prime} \leq \mathbf{x}^{*}, \mathbf{y}^{\prime} \geq \mathbf{y}^{*}$, and $\left(\mathbf{x}^{\prime}, \mathbf{y}^{\prime}\right) \neq\left(\mathbf{x}^{*}, \mathbf{y}^{*}\right)$. Without loss of generality, let $\left(\mathbf{x}^{\prime}, \mathbf{y}^{\prime}\right)$ satisfy (1) with some vector $\boldsymbol{\lambda}^{\prime}$ such that all input and output inequalities in (1) are satisfied as equalities, i.e., without slacks. Then $\left(\mathbf{x}^{\prime}, \mathbf{y}^{\prime}\right)$ satisfies (7) with $\boldsymbol{\lambda}=\boldsymbol{\lambda}^{\prime}$ and $\boldsymbol{\mu}=\boldsymbol{\nu}=\mathbf{0}$. Therefore, $\left(\mathbf{x}^{\prime}, \mathbf{y}^{\prime}\right) \in \mathcal{T}_{\text {VRS }}^{\mathrm{HD}}$, which contradicts the assumption that $\left(\mathbf{x}^{*}, \mathbf{y}^{*}\right) \in \mathcal{E}_{\mathrm{HD}}^{*}$. Therefore, $\left(\mathbf{x}^{*}, \mathbf{y}^{*}\right) \in \mathcal{E}_{\mathrm{VRS}}^{*}$.

The proof of statement (ii) is similar to the second part of the proof of statement (i) and is not given.

Proof of Theorem 4.7. The part (i) follows from the fact that the statements of technologies $\mathcal{T}_{\text {NIRS }}^{\mathrm{HD}}$ and $\mathcal{T}_{\text {CRS }}^{\mathrm{HD}}$ are obtained from (7) by replacing the equality $\mathbf{1}^{T} \boldsymbol{\lambda}=1$ by the inequality $\mathbf{1}^{T} \boldsymbol{\lambda} \leq 1$ and by removing this condition, respectively.

Let us prove part (ii). Its first embedding is established by (4) and needs no further proof. Let $\operatorname{DMU}(\mathbf{x}, \mathbf{y}) \in \mathcal{T}_{\text {NIRS }}^{\text {HD }}$. This DMU satisfies (7) in which the equality $\mathbf{1}^{T} \boldsymbol{\lambda}=1$ is changed to the inequality $\mathbf{1}^{T} \boldsymbol{\lambda} \leq 1$, with some vectors $\boldsymbol{\lambda}^{\prime}, \boldsymbol{\mu}^{\prime}$ and $\boldsymbol{\nu}^{\prime}$. Then it also satisfies (with the same $\boldsymbol{\lambda}^{\prime}, \boldsymbol{\mu}^{\prime}$ and $\boldsymbol{\nu}^{\prime}$ ) these conditions in which we replace the equalities for the input vector $\mathbf{x}^{W}$ and output vector $\mathbf{y}^{W}$ by the non-strict inequalities " $\leq$ " and " $\geq$ ", respectively. Then $\operatorname{DMU}(\mathbf{x}, \mathbf{y})$ satisfies the resulting conditions with $\boldsymbol{\lambda}^{\prime}$ and $\boldsymbol{\mu}=\mathbf{0}$ and $\boldsymbol{\nu}=\mathbf{0}$. (Note that replacing $\boldsymbol{\mu}^{\prime}$ by $\mathbf{0}$ makes the left-hand side of the inequality for $\mathbf{x}^{W}$ smaller, and a similar replacement of $\boldsymbol{\nu}^{\prime}$ by $\mathbf{0}$ makes the left-hand side of the inequality for $\mathbf{y}^{W}$ larger, keeping both inequalities satisfied.) Therefore, DMU (x,y) satisfies the conditions of the conventional strongly disposable NIRS technology $\mathcal{T}_{\text {NIRS }}$, and the second embedding in part (ii) follows. The proof of the final embedding in (ii) is similar and is not given.

\section{Appendix B. Data generating process}

To generate each school in a sample, we first randomly generate a provisional efficient school $\left(\tilde{x}_{1}, \tilde{x}_{2}, \tilde{y}_{1}, \tilde{y}_{2}\right)$ that satisfies equality (21). We subsequently introduce random inefficiency to its outputs. The resulting school enters the sample.

More specifically, we first generate the number of teachers $\tilde{x}_{1}$ by random draw from the uniform distribution $U[30,100]$. We then generate the school expenditure as follows:

$$
\tilde{x}_{2}=15 \pi \tilde{x}_{1},
$$

where $\pi$ is drawn from $U[0.8,1.5]$. This reflects the fact that larger schools with more teachers are expected to have higher expenditure, but the link is not deterministic. For example, some schools with shortage of teachers may compensate for this by higher expenditure on class assistants.

We subsequently calculate the overall resource of the school as the last term $R=$ $20 \tilde{x}_{1}^{0.8} \tilde{x}_{2}^{0.2}$ in (21). We assume that, at any school, no more than $R / 2$ is spent on pupils with special needs. By formula (21), each pupil with special needs (who is accounted twice, in $y_{1}$ and $y_{2}$ ), requires the total resource equal to the sum of coefficients $1+0.5=1.5$. This 
implies that, given the overall resource $R$, the number of pupils with special needs does not exceed $(R / 2) / 1.5=R / 3$. Consequently, we randomly generate $\tilde{y}_{2}$ from $U[0, R / 3]$.

Using equation (21), we calculate the number of pupils who do not have special needs as $\tilde{z}=R-1.5 \tilde{y}_{2}$. We calculate the overall number of pupils as $\tilde{y}_{1}=\tilde{z}+\tilde{y}_{2}$.

The described procedure produces a random school $\left(\tilde{x}_{1}, \tilde{x}_{2}, \tilde{y}_{1}, \tilde{y}_{2}\right)$ whose components satisfy equality (21). This school is located on the production frontier and is therefore efficient. Next, we introduce inefficiency. We draw the output radial efficiency $\varepsilon$ from $U[0.5,1]$ and multiply both outputs $\tilde{y}_{1}$ and $\tilde{y}_{2}$ by this number. The resulting school $\left(\tilde{x}_{1}, \tilde{x}_{2}, \varepsilon \tilde{y}_{1}, \varepsilon \tilde{y}_{2}\right)$ has the theoretical output radial efficiency equal to $\varepsilon$. This school enters the sample.

The described process is repeated to obtain the required number of schools in the sample. 


\section{References}

Afriat, S. N. (1972). Efficiency estimation of production functions. International Economic Review, 13(3), 568-598.

Banker, R. D. (1984). Estimating most productive scale size using data envelopment analysis. European Journal of Operational Research, 17(1), 35-44.

Banker, R. D., Charnes, A., \& Cooper, W. W. (1984). Some models for estimating technical and scale efficiencies in data envelopment analysis. Management Science, 30(9), 1078-1092.

Banker, R. D., Cooper, W. W., Seiford, L. M., \& Zhu, J. (2011). Returns to scale in DEA. In W. W. Cooper, L. M, Seiford, \& J. Zhu (Eds.), Handbook on data envelopment analysis. (2nd ed.). (pp. 41-70). New York: Springer Science + Busines Media.

Banker, R. D., \& Morey, R. C. (1986). Efficiency analysis for exogenously fixed inputs and outputs. Operations Research, 34(4), 513-521.

Banker, R. D., \& Thrall, R. M. (1992). Estimation of returns to scale using data envelopment analysis. European Journal of Operational Research, 62(1), 74-84.

Briec, W., Kerstens, K., Leleu, H., \& Vanden Eeckaut, P. (2000). Returns to scale on nonparametric deterministic technologies: Simplifying goodness-of-fit methods using operations on technologies. Journal of Productivity Analysis, 14(3), 267-274.

Byrnes, P., Färe, R., Grosskopf, S., \& Lovell, C. A. K. (1988). The effect of unions on productivity: US surface mining of coal. Management Science, 34(9), 1037-1053.

Cesaroni, G., Kerstens, K., \& Van de Woestyne, I. (2017). Global and local scale characteristics in convex and nonconvex nonparametric technologies: A first empirical exploration. European Journal of Operational Research, 259(2), 576-586.

Chambers, R. G., Chung, Y., \& Färe, R. (1998). Profit, directional distance functions, and Nerlovian efficiency. Journal of Optimization Theory and Applications, 98(2), 351-364.

Charnes, A., Cooper, W. W., \& Rhodes, E. (1978). Measuring the efficiency of decision making units. European Journal of Operational Research, 2(6), 429-444.

Deprins, D., Simar, L., \& Tulkens, H. (1984). Measuring labor-efficiency in post offices. In M. Marchand, P. Pestieau, H. Tulkens (Eds.), The performance of public enterprises: concepts and measurements (pp. 243267). Amsterdam: North-Holland.

Dervaux, B., Kerstens, K., \& Vanden Eeckaut, P. (1998). Radial and nonradial static efficiency decompositions: A focus on congestion measurement. Transportation Research. Part B: Methodological, 32(5), 299-312.

Färe, R., \& Grosskopf, S. (1983). Measuring congestion in production. Zeitschrift für Nationalökonomie / Journal of Economics, 43(3), 257-271.

Färe, R., \& Grosskopf, S. (2004). New directions: Efficiency and productivity. Dordrecht: Kluwer Academic Publishers.

Färe, R., Grosskopf, S., \& Logan, J. (1983). The relative efficiency of Illinois electric utilities. Resources and Energy, 5(4), 349-367.

Färe, R., Grosskopf, S., \& Lovell, C. A. K. (1983). The structure of technical efficiency. The Scandinavian Journal of Economics, 85(2), 181-190.

Färe, R., Grosskopf, S., \& Lovell, C. A. K. (1985). The measurement of efficiency of production. Boston: Kluwer Academic Publishers.

Färe, R., Grosskopf, S., \& Lovell, C. A. K. (1987). Nonparametric disposability tests. Zeitschrift für Nationalökonomie / Journal of Economics, 47(1), 77-85.

Färe, R., Grosskopf, S., \& Lovell, C. A. K. (1994). Production frontiers. Cambridge: Cambridge University Press.

Färe, R., \& Primont, D. (1995). Multi-output production and duality: Theory and applications. Boston: Kluwer Academic Publishers.

Førsund, F. R. (1996). On the calculation of scale elasticity in DEA models. Journal of Productivity Analysis, $7(2-3), 283-302$.

Førsund, F. R., \& Hjalmarsson, L. (2004). Calculating scale elasticity in DEA models. Journal of the Operational Research Society, 55(10), 1023-1038.

Førsund, F. R., \& Sarafoglou, N. (2002). On the origins of data envelopment analysis. Journal of Productivity Analysis, 17(1), 23-40.

Grosskopf, S. (1986). The role of the reference technology in measuring productive efficiency. The Economic Journal, 96(382), 499-513. 
Hadjicostas, P., \& Soteriou, A. C. (2006). One-sided elasticities and technical efficiency in multi-output production: A theoretical framework. European Journal of Operational Research, 168(2), 425-449.

Kerstens, K., \& Vanden Eeckaut, P. (1999). Estimating returns to scale using non-parametric deterministic technologies: A new method based on goodness-of-fit. European Journal of Operational Research, 113(1), 206-214.

Kuosmanen, T. (2005). Weak disposability in nonparametric productivity analysis with undesirable outputs. American Journal of Agricultural Economics, 87(4), 1077-1082.

Mehdiloozad, M., \& Podinovski, V. V. (2018). Nonparametric production technologies with weakly disposable inputs. European Journal of Operational Research, 266(1), 247-258.

Pham, M. D., \& Zelenyuk, V. (2018). Weak disposability in nonparametric production analysis: A new taxonomy of reference technology sets. European Journal of Operational Research, 274(1), 186-198.

Podinovski, V. V. (2017). Returns to scale in convex production technologies. European Journal of Operational Research, 258(3), 970-982.

Podinovski, V. V., \& Bouzdine-Chameeva, T. (2019). Cone extensions of polyhedral production technologies. European Journal of Operational Research, doi.: 10.1016/j.ejor.2019.01.031.

Podinovski, V. V., Chambers, R. G., Atici, K. B., \& Deineko, I. D. (2016). Marginal values and returns to scale for nonparametric production frontiers. Operations Research, 64 (1), 236-250.

Podinovski, V. V., \& Førsund, F. R. (2010). Differential characteristics of efficient frontiers in data envelopment analysis. Operations Research, 58(6), 1743-1754.

Podinovski, V. V., \& Kuosmanen, T. (2011). Modelling weak disposability in data envelopment analysis under relaxed convexity assumptions. European Journal of Operational Research, 211(3), 577-585.

Sahoo, B. K., \& Tone, K. (2015). Scale elasticity in non-parametric DEA approach. In J. Zhu (Ed.), Data envelopment analysis: A handbook of models and methods (pp. 269-290). New York: Springer Science + Busines Media.

Shephard, R. W. (1974a). Indirect production functions. Mathematical Systems in Economics No. 10. Meisenheim am Glan: Anton Hain.

Shephard, R. W. (1974b). Semi-homogeneous production functions and scaling of production. In W. Eichhorn, R. Henn, O. Opitz, R. W. Shephard (Eds.), Production theory (pp. 253-285). New York: SpringerVerlag.

Sueyoshi, T., \& Goto, M. (2012). Weak and strong disposability vs. natural and managerial disposability in DEA environmental assessment: Comparison between Japanese electric power industry and manufacturing industries. Energy Economics, 34(3), 686-699.

Tone, K. (2001). A slacks-based measure of efficiency in data envelopment analysis. European Journal of Operational Research, 130(3), 498-509.

Tone, K., \& Tsutsui, M. (2011). Applying an efficiency measure of desirable and undesirable outputs in DEA to U.S. electric utilities. Journal of CENTRUM Cathedra, 4(2), 236-249.

Zelenyuk, V. (2013). A scale elasticity measure for directional distance function and its dual: Theory and DEA estimation. European Journal of Operational Research, 228(3), 592-600. 